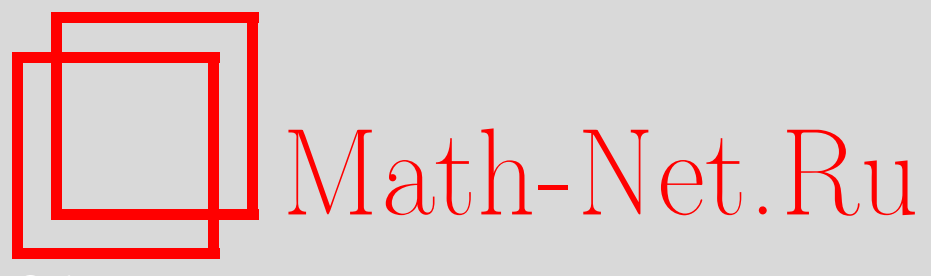

Н. Х. Ибрагимов, Е. Д. Авдонина, Нелинейная самосопряженность, законы сохранения и построение решений уравнений в частных производных с помощью законов сохранения, УМН, 2013, том 68, выпуск 5, 111-146

DOI: https://doi.org/10.4213/rm9536

Использование Общероссийского математического портала Math-Net.Ru подразумевает, что вы прочитали и согласны с пользовательским соглашением http://www.mathnet.ru/rus/agreement

Параметры загрузки:

IP: 34.239 .49 .27

26 апреля 2023 г., 18:11:59

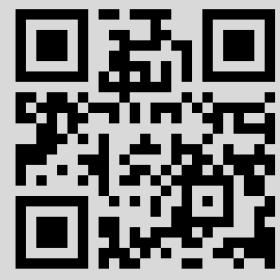




\title{
Нелинейная самосопряженность, законы сохранения и построение решений уравнений в частных производных с помощью законов сохранения
}

\author{
Н. Х. Ибрагимов, Е. Д. Авдонина
}

Метод нелинейной самосопряженности, развитый недавно первым автором, обобщает теорему Нётер. Этот новый метод значительно расширяет возможности построения законов сохранения, ассоциированных с симметриями, так как не требует существования лагранжиана. В частности, этот метод применим к любым линейным уравнениям и к любым нелинейным уравнениям, обладающим хотя бы одним локальным законом сохранения. В настоящей статье дается краткий обзор результатов о законах сохранения, полученных методом нелинейной самосопряженности и опубликованных в основном в наших недавних препринтах, а также излагается способ построения точных решений систем дифференциальных уравнений в частных производных с помощью законов сохранения. Решения, полученные методом законов сохранения, в большинстве случаев не могут быть найдены как инвариантные или частично инвариантные решения.

Библиография: 23 названия.

Ключевые слова: дифференциальные уравнения, нелинейная самосопряженность, законы сохранения, точные решения.

DOI: $10.4213 / \mathrm{rm} 9536$

\section{СОДЕРЖАНИЕ}

Введение .............................................. 112

Глава 1. Нелинейная самосопряженность и законы сохранения . . . . . . . 113

1.1. Определение и свойства нелинейной самосопряженности . . . . . . . 113

1.1.1. Обозначения ................................. 114

1.1.2. Сопряженные уравнения для систем нелинейных дифферен-

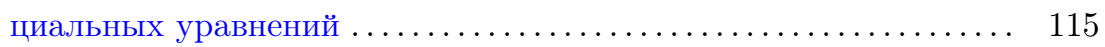

1.1.3. Строгая самосопряженность ................... 116

1.1.4. Определение нелинейной самосопряженности.............. 116

1.1.5. Свойства нелинейной самосопряженности .............. 118

Работа выполнена при поддержке Правительства РФ (дог. № 11.G34.31.0042).

(C) Н. Х. ИвраГимов, Е. Д. Авдонина, 2013 
1.2. Построение законов сохранения ......................... 119

1.2.1. Основная теорема ............................ 119

1.2.2. Законы сохранения, зависящие от времени, для уравнения Ка-

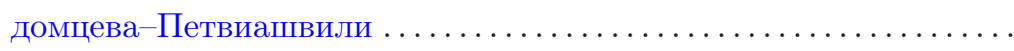

1.2.3. Модель распространения ультракоротких световых импульсов

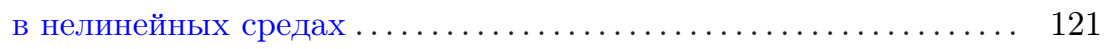

Глава 2. Построение решений методом законов сохранения . . . . . . . . . 123

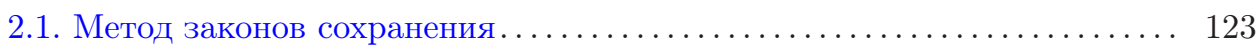

2.1.1. Обыкновенные дифференциальные уравнения............. 123

2.1.2. Системы уравнений в частных производных ............. 124

2.2. Применение к газу Чаплыгина .......................... 125

2.2.1. Локальные и нелокальные симметрии .................. 125

2.2.2. Локальные законы сохранения ..................... 126

2.2.3. Нелокальные законы сохранения.................. 127

2.2.4. Решения, полученные из закона сохранения массы ......... 127

2.2.5. Решения, полученные из закона сохранения количества дви-

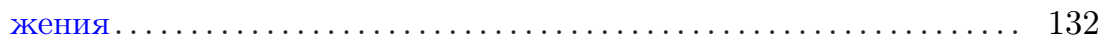

2.3. Одномерный политропный газ ...................... 134

2.4. Нелинейная диффузия в анизотропных средах ................ 134

2.4.1. Законы сохранения двумерной модели с источником специаль-

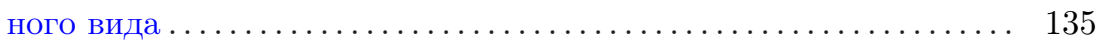

2.4.2. Построение точных решений ..................... 136

2.4.3. Самосопряженность и нестационарные решения трехмерной модели с линейным источником...................

Глава 3. Приближенная самосопряженность и приближенные законы со-

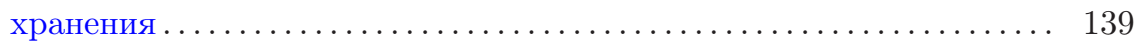

3.1. Введение ........................................ 139

3.2. Приближенно сопряженное уравнение .................... 140

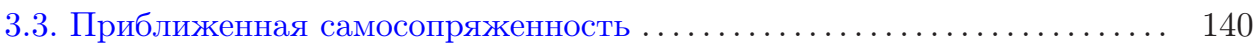

3.4. Приближенные симметрии . . . . . . . . . . . . . . . . . . . . 141

3.5. Приближенные законы сохранения.......................... 142

Список литературы ......................................... 144

\section{Введение}

Групповой анализ дифференциальных уравнений как новая ветвь науки был сформирован в работах выдающегося норвежского математика Софуса Ли [1]. Он первым использовал концепцию групп для интегрирования обыкновенных дифференциальных уравнений произвольного порядка. Разработанный им аппарат применим также к уравнениям в частных производных и является единственным универсальным методом, позволяющим решать нелинейные дифференциальные уравнения аналитически. 
Ли также ввел понятие инвариантных решений дифференциальных уравнений в частных производных. Это понятие было в дальнейшем развито и расширено введением понятия частично инвариантных решений Л. В. Овсянниковым [2], [3]. Такие решения играют существенную роль в современных исследованиях точных решений нелинейных математических моделей в естественных и инженерных науках.

Известная теорема Нётер позволяет использовать симметрии для построения законов сохранения дифференциальных уравнений, обладающих лагранжианом, при дополнительном условии, что рассматриваемые симметрии оставляют инвариантным вариационный интеграл.

Метод нелинейной самосопряженности, разработанный в статьях [4]-[6], обобщает теорему Нётер. Этот новый метод значительно расширяет возможности построения законов сохранения, ассоциированных с симметриями, так как не требует существования лагранжиана. В частности, этот метод применим к любым линейным уравнениям, а также к любым нелинейным уравнениям, обладаюшим хотя бы одним локальным законом сохранения (т. е. законом сохранения, который может включать конечное число производных зависимых переменных, но не содержит нелокальных переменных типа интегралов от зависимых переменных). Кроме того, метод нелинейной самосопряженности, в отличие от теоремы Нётер, не требует, чтобы число уравнений в системе уравнений было равно числу зависимых переменных. Число уравнений может быть любым. Например, система может быть переопределенной - как система уравнений Максвелла, или незамкнутой - как уравнения газовой динамики с неизвестным уравнением состояния.

Недавно было показано [7], что знание законов сохранения позволяет построить точные решения систем уравнений в частных производных, отличные от инвариантно-групповых решений.

В последнее время новый метод применялся к различным нелинейным математическим моделям. Например, в наших работах [8]-[11] этим методом исследовались различные модели диффузии в анизотропных средах и течения политропного газа.

В настоящей статье дается краткий обзор упомянутых выше работ, а также приводятся новые результаты о законах сохранения, полученные методом нелинейной самосопряженности, вместе с определяемыми этими законами сохранения точными решениями.

\section{Глава 1. Нелинейная самосопряженность и законы сохранения}

\section{1. Определение и свойства нелинейной самосопряженности}

Понятие самосопряженности нелинейных уравнений было сначала введено в смысле строгой самосопряженности [4] для построения законов сохранения, ассоциированных с симметриями дифференциальных уравнений. Затем возможности нового метода были расширены путем введения понятия квазисамосопряженности (см. [5; раздел 1.6]). Наконец, в работе [5] было введено 
общее понятие нелинейной самосопряженности. Оно включает в себя, наряду с обычной линейной самосопряженностью, понятия строгой самосопряженности и квазисамосопряженности. Кроме того, все линейные уравнения обладают свойством нелинейной самосопряженности.

Было также показано, что уравнения, обладающие нелинейной самосопряженностью, могут быть записаны эквивалентным образом в строго самосопряженном виде с помощью использования соответствующих множителей. Например, линейное уравнение теплопроводности $u_{t}-\Delta u=0$ становится строго самосопряженным, если переписать его в нелинейном виде $\left(u_{t}-\Delta u\right) / u=0$.

1.1.1. Обозначения. Пусть $x=\left(x^{1}, \ldots, x^{n}\right)$ - независимые переменные. Рассмотрим зависимые переменные $u=\left(u^{1}, \ldots, u^{m}\right)$ и $v=\left(v^{1}, \ldots, v^{m}\right)$ с соответствующими частными производными

$$
\begin{array}{llll}
u_{(1)}=\left\{u_{i}^{\alpha}\right\}, & u_{(2)}=\left\{u_{i j}^{\alpha}\right\}, & \ldots, & u_{(s)}=\left\{u_{i_{1} \ldots i_{s}}^{\alpha}\right\}, \\
v_{(1)}=\left\{v_{i}^{\alpha}\right\}, & v_{(2)}=\left\{v_{i j}^{\alpha}\right\}, \quad \ldots, & v_{(s)}=\left\{v_{i_{1} \ldots i_{s}}^{\alpha}\right\},
\end{array}
$$

где

$$
\begin{array}{llll}
u_{i}^{\alpha}=D_{i}\left(u^{\alpha}\right), & u_{i j}^{\alpha}=D_{i} D_{j}\left(u^{\alpha}\right), \quad \ldots, & u_{i_{1} \ldots i_{s}}^{\alpha}=D_{i_{1}} \cdots D_{i_{s}}\left(u^{\alpha}\right), \\
v_{i}^{\alpha}=D_{i}\left(v^{\alpha}\right), & v_{i j}^{\alpha}=D_{i} D_{j}\left(v^{\alpha}\right), & \ldots, & v_{i_{1} \ldots i_{s}}^{\alpha}=D_{i_{1}} \cdots D_{i_{s}}\left(v^{\alpha}\right) .
\end{array}
$$

В этих формулах $D_{i}$ обозначает оператор полного дифферениирования

$$
D_{i}=\frac{\partial}{\partial x^{i}}+u_{i}^{\alpha} \frac{\partial}{\partial u^{\alpha}}+v_{i}^{\alpha} \frac{\partial}{\partial v^{\alpha}}+u_{i j}^{\alpha} \frac{\partial}{\partial u_{j}^{\alpha}}+v_{i j}^{\alpha} \frac{\partial}{\partial v_{j}^{\alpha}}+u_{i j k}^{\alpha} \frac{\partial}{\partial u_{j k}^{\alpha}}+v_{i j k}^{\alpha} \frac{\partial}{\partial v_{j k}^{\alpha}}+\cdots ;
$$

здесь и далее используется общепринятое правило суммирования по повторяющимся индексам.

Законом сохранения для системы дифференциальных уравнений в частных производных называется уравнение

$$
D_{i}\left(C^{i}\right)=0
$$

которое должно выполняться на всех решениях рассматриваемой системы уравнений в частных производных. По повторяющемуся индексу $i$ предполагается суммирование от 1 до $n$. Величины $C^{i}$ являются гладкими функциями

$$
C^{i}=C^{i}\left(x, u, u_{(1)}, \ldots\right), \quad i=1, \ldots, n,
$$

и могут содержать производные $u_{(1)}, \ldots$ до любого конечного порядка. Вектор

$$
C=\left(C^{1}, \ldots, C^{n}\right)
$$

с компонентами (1.2), удовлетворяющими уравнению (1.1), называется сохраняющимся вектором для данного уравнения в частных производных.

Закон сохранения (1.1) с сохраняющимся вектором указанного выше вида называется локальным законом сохранения. Для некоторых уравнений компоненты (1.2) сохраняющегося вектора могут зависеть от дополнительных переменных, например, от так называемых нелокальных переменных. Тогда мы 
имеем дело с нелокальными законами сохранения. Газ Чаплыгина, рассматриваемый далее в п. 2.2, имеет нелокальные законы сохранения.

Читатель, интересующийся дальнейшими подробностями о приведенных выше понятиях оператора полного дифференцирования, законов сохранения и нелокальных переменных, может обратиться, например, к монографии [12].

\subsection{2. Сопряженные уравнения для систем нелинейных дифферен-} циальных уравнений. Рассматривается система $m$ дифференциальных уравнений (линейных или нелинейных)

$$
F_{\alpha}\left(x, u, u_{(1)}, \ldots, u_{(s)}\right)=0, \quad \alpha=1, \ldots, m,
$$

c $m$ зависимыми переменными.

Всюду в дальнейшем предполагается, что система (1.4) регулярно задана в терминологии, принятой, например, в [3; гл. 1] (см. также [13]), или имеет максимальный ранг - в смысле определения, данного в [14; гл. 2]. Это означает, что матрица Якоби функций $F_{\alpha}$ по всем их аргументам, т. е. матрица

$$
J=\left\|\frac{\partial F_{\alpha}}{\partial x}, \frac{\partial F_{\alpha}}{\partial u}, \frac{\partial F_{\alpha}}{\partial u_{(1)}}, \ldots, \frac{\partial F_{\alpha}}{\partial u_{(s)}}\right\|,
$$

имеет ранг, равный $m$ (числу уравнений (1.4)) во всех точках, удовлетворяющих системе (1.4). Например, уравнение Кортевега-де Фриза (КдФ) $u_{t}-u u_{x}-$ $u_{x x x}=0$ регулярно задано, а то же уравнение, записанное в виде $\left(u_{t}-u u_{x}-\right.$ $\left.u_{x x x}\right)^{2}=0$, не является регулярно заданным, так как тогда ранг соответствующей матрицы Якоби обращается в нуль на решениях уравнения КдФ.

Сопряжсенные уравнения к уравнениям (1.4) записываются в виде

$$
F_{\alpha}^{*}\left(x, u, v, u_{(1)}, v_{(1)}, \ldots, u_{(s)}, v_{(s)}\right)=0, \quad \alpha=1, \ldots, m,
$$

где сопряженный оператор $F_{\alpha}^{*}$ определяется формулой

$$
F_{\alpha}^{*}\left(x, u, v, u_{(1)}, v_{(1)}, \ldots, u_{(s)}, v_{(s)}\right)=\frac{\delta \mathscr{L}}{\delta u^{\alpha}} .
$$

Здесь $\mathscr{L}$ - формалъный лагранжиан для уравнений (1.4), определяемый формулой

$$
\mathscr{L}=\sum_{\beta=1}^{m} v^{\beta} F_{\beta}\left(x, u, u_{(1)}, \ldots, u_{(s)}\right),
$$

a $\delta / \delta u^{\alpha}-$ вариационная производная

$$
\frac{\delta}{\delta u^{\alpha}}=\frac{\partial}{\partial u^{\alpha}}+\sum_{s=1}^{\infty}(-1)^{s} D_{i_{1}} \cdots D_{i_{s}} \frac{\partial}{\partial u_{i_{1} \ldots i_{s}}^{\alpha}} .
$$

Для линейного уравнения $L[u]=0$ сопряженный оператор, определяемый формулой (1.6), совпадает с сопряженным оператором $L^{*}[v]$ в классическом определении, который задается уравнением $v L[u]-u L^{*}[v]=D_{i}\left(p^{i}\right)$.

Сопряженность линейных операторов $L$ является симметричным отношением, а именно, $\left(L^{*}\right)^{*}=L$. Нелинейные уравнения не обладают этим свойством, так что, вообще говоря, $\left(F^{*}\right)^{*}$ не совпадает с $F$. 
1.1.3. Строгая самосопряженность. Линейный оператор $L$ называется самосопряженным, если $L^{*}=L$. Тогда говорят также, что уравнение $L[u]=0$ самосопряженно. Таким образом, самосопряженность линейного уравнения $L[u]=0$ означает, что сопряженное уравнение $L^{*}[v]=0$ совпадает с $L[u]=0$ после подстановки

$$
v=u \text {. }
$$

Данное свойство переносится на нелинейные уравнения в следующем смысле строгой самосопряженности.

ОПРЕДЕЛЕниЕ 1.1. Уравнение (1.4) называется строго самосопряженным, если после подстановки (1.8) сопряженное уравнение (1.5) становится эквивалентным первоначальному уравнению (1.4).

Например, уравнение КдФ

$$
u_{t}=u_{x x x}+u u_{x}
$$

является строго самосопряженным. Действительно, его сопряженное уравнение (1.5) имеет вид

$$
v_{t}=v_{x x x}+u v_{x}
$$

и совпадает с уравнением КдФ после подстановки $v=u$.

Понятие квазисамосопряженности является обобщением определения $1.1 \mathrm{c}$ помощью замены (1.8) подстановкой вида

$$
v=\varphi(u), \quad \varphi^{\prime}(u) \neq 0 .
$$

Таким образом, уравнение (1.4) является квазисамосопряженным, если сопряженное уравнение (1.5) становится эквивалентным уравнению (1.4) после подстановки (1.9). В качестве примера рассмотрим уравнение

$$
u_{t}-u^{2} u_{x x}=0
$$

описывающее нелинейную теплопроводность в твердом водороде. Его сопряженное уравнение (1.5) имеет вид

$$
v_{t}+4 u v u_{x x}+u^{2} v_{x x}+4 u u_{x} v_{x}+2 v u_{x}^{2}=0 .
$$

Оно становится эквивалентным уравнению (1.10) не после подстановки (1.8), а после следующей подстановки вида (1.9):

$$
v=u^{-2} \text {. }
$$

1.1.4. Определение нелинейной самосопряженности. Понятие нелинейной самосопряженности будет играть существенную роль в дальнейшем, начиная с п. 1.2.1, где дается общая формула (1.21) для построения законов сохранения. В основе этой формулы лежит формальный лагранжиан $\mathscr{L}$. Определение (1.7) формального лагранжиана $\mathscr{L}$ показывает, что вектор (1.21) включает в себя "нефизическую" переменную $v$. Поэтому закон сохранения (1.1), 
$D_{i}\left(C^{i}\right)=0$, выполняется, если мы принимаем во внимание не только уравнения (1.4), но и сопряженные уравнения (1.5). Если же система (1.4) является квазисамосопряженной (в частности, строго самосопряженной), лишнюю переменную $v$ можно исключить с помощью подстановки (1.9) и получить закон сохранения для уравнений (1.4).

Однако квазисамосопряженность не единственный случай, когда переменные $v$ могут быть исключены из сохраняющегося вектора (1.21). Заметим в первую очередь, что мы можем ослабить условие $\varphi^{\prime}(u) \neq 0$ в $(1.9)$, поскольку оно используется лишь с целью обеспечения эквивалентности уравнения (1.5) уравнению (1.4) после исключения $v$ подстановкой $v=\varphi(u)$. При построении законов сохранения важно лишь то, что $v$ не тождественно равно нулю, поскольку в противном случае $\mathscr{L}=0$ и $(1.21)$ дает тривиальный вектор $C^{i}=0$. Поэтому мы можем заменить условие $\varphi^{\prime}(u) \neq 0(1.9)$ более слабым условием $\varphi(u) \neq 0$. Кроме того, подстановку (1.9) можно заменить более общей подстановкой, где $\varphi$ содержит независимые переменные $x$ и зависимые переменные $u$ вместе с их частными производными любого конечного порядка.

Итак, рассматривается общая дифференциальная подстановка (см. [12])

$$
v^{\alpha}=\varphi^{\alpha}\left(x, u, u_{(1)}, \ldots\right), \quad \alpha=1, \ldots, m
$$

при условии, что не все $\varphi^{\alpha}$ тождественно равны нулю.

ОПРЕДЕЛЕНИЕ 1.2. Система (1.4) называется нелинейно самосопряженной, если сопряженная система (1.5) удовлетворяется для всех решений уравнений (1.4) после некоторой подстановки (1.12).

ЗАМЕчАНИЕ 1.1. В практических приложениях часто используются подстановки (1.12), в которые не входят производные, т. е. подстановки вида

$$
v^{\alpha}=\varphi^{\alpha}(x, u), \quad \alpha=1, \ldots, m
$$

Тогда для регулярно заданной системы (1.4) определение 1.2 нелинейной самосопряженности равносильно выполнению уравнения

$$
F_{\alpha}^{*}\left(x, u, \varphi(x, u), \ldots, u_{(s)}, \varphi_{(s)}\right)=\lambda_{\alpha}^{\beta} F_{\beta}\left(x, u, \ldots, u_{(s)}\right), \quad \alpha=1, \ldots, m,
$$

где $\lambda_{\alpha}^{\beta}$ - неопределенные переменные коэффициенты, не обращающиеся в бесконечность на решениях уравнений (1.4). Здесь $\varphi$ есть $m$-мерный вектор

$$
\varphi=\left(\varphi^{1}, \ldots, \varphi^{m}\right)
$$

а $\varphi_{(\sigma)}$ суть его производные:

$$
\varphi_{(\sigma)}=\left\{D_{i_{1}} \cdots D_{i_{\sigma}}\left(\varphi^{\alpha}(x, u)\right)\right\}, \quad \sigma=1, \ldots, s .
$$




\subsection{5. Свойства нелинейной самосопряженности.}

ПРЕДЛОЖЕНИЕ 1.1. Любое линейное уравнение является нелинейно самосопряженным.

ДокАЗАтельство. Это следует из того факта, что уравнение $L^{*}[v]=0$, сопряженное линейному уравнению $L[u]=0$, не содержит переменную $u$. Поэтому любое ненулевое решение $v=\varphi(x)$ сопряженного уравнения дает векторную функцию (1.13), которая не зависит от $u$ и поэтому удовлетворяет требованию определения 1.2 .

ПрЕдЛОжЕниЕ 1.2. Если система дифференииальных уравнений (1.4) имеет хотя бы один локальный закон сохранения, то она является нелинейно самосопряженной [5; теорема 8.1].

ДокАЗАТЕЛьство. Пусть регулярно заданная система (1.4) имеет локальный закон сохранения (1.1). Для простоты предположим, что максимальный порядок производных, входящих в компоненты сохраняющегося вектора, ниже, чем порядок производных в уравнениях системы (1.4). Тогда условие того, что уравнение (1.1) выполняется на всех решениях системы (1.4), можно записать в виде

$$
D_{i}\left(C^{i}\right)=\mu^{\beta} F_{\beta}\left(x, u, u_{(1)}, \ldots, u_{(s)}\right)
$$

с некоторыми коэффициентами $\mu^{\alpha}=\mu^{\alpha}\left(x, u, u_{(1)}, \ldots, u_{(s)}\right)$. Теперь можно воспользоваться хорошо известным результатом о том, что вариационная производная дивергентного выражения тождественно обращается в нуль ${ }^{1}$. Поэтому из (1.15) следует выполнение уравнения

$$
\frac{\delta}{\delta u^{\alpha}}\left[\mu^{\beta} F_{\beta}\left(x, u, u_{(1)}, \ldots, u_{(s)}\right)\right]=0 .
$$

Сравнение уравнений (1.16) и (1.6) показывает, что условие определения 1.2 нелинейной самосопряженности выполняется с подстановкой (1.12) вида

$$
v^{\alpha}=\mu^{\alpha}\left(x, u, u_{(1)}, \ldots, u_{(s)}\right) .
$$

Это завершает доказательство.

ЗАмЕЧАнИЕ 1.2. В предложении 1.2 условие локалъности законов сохранения существенно. А именно, в [5; примеры 12.2 и 12.3], показано, что вариационные производные дивергентных выражений $D_{i}\left(C^{i}\right)$ с нелокальными $C^{i}$ не обращаются в нуль.

ПРЕДЛОЖЕниЕ 1.3. Уравнение (1.4) с одной зависимой переменной $(m=1)$ является нелинейно самосопряженным, если и только если оно становится строго самосопряженным (см. определение 1.1), будучи переписано в эквивалентной форме

$$
\mu(x, u) F\left(x, u, u_{(1)}, \ldots, u_{(s)}\right)=0
$$

с подходящим множителем $\mu(x, u) \neq 0$. В частности, любое линейное уравнение можно сделать самосопряженным в смысле определения 1.1.

\footnotetext{
1Этот результат кратко упоминается в книге [15; т. I, гл. 5, §3.5]. Более подробное изложение можно найти, например, в [16; раздел 8.4.1].
} 
ДокАЗАтЕльство. Вычисления показывают (см. [5]), что существует следующее соотношение между множителем $\mu(x, u)$ в $(1.18)$ и подстановкой $(1.13)$ :

$$
\varphi(x, u)=u \mu(x, u) .
$$

Таким образом, если уравнение (1.4) нелинейно самосопряженно при подстановке (1.13), то уравнение (1.18) строго самосопряженно с множителем $\mu$, определенным уравнением (1.19), и наоборот.

\section{2. Построение законов сохранения}

1.2.1. Основная теорема. Доказательство следующего утверждения можно найти в [4].

ТЕорема 1.1. Любая инфинитезимальная симметрия (точечная симметрия Ли, Ли-Бэклунда, нелокальная)

$$
X=\xi^{i}\left(x, u, u_{(1)}, \ldots\right) \frac{\partial}{\partial x^{i}}+\eta^{\alpha}\left(x, u, u_{(1)}, \ldots\right) \frac{\partial}{\partial u^{\alpha}}
$$

нелинейно самосопряженной системы (1.4) ведет $к$ закону сохранения (1.1) для этой системь:

$$
\left[D_{i}\left(C^{i}\right)\right]_{(1.4)}=0
$$

(здесь нижний индекс (1.4) означает, что выражение в квадратных скобках рассматривается на решениях системы (1.4)). Компоненты сохраняющегося вектора задаются формулами

$$
\begin{aligned}
C^{i}=\xi^{i} \mathscr{L} & +W^{\alpha}\left[\frac{\partial \mathscr{L}}{\partial u_{i}^{\alpha}}-D_{j}\left(\frac{\partial \mathscr{L}}{\partial u_{i j}^{\alpha}}\right)+D_{j} D_{k}\left(\frac{\partial \mathscr{L}}{\partial u_{i j k}^{\alpha}}\right)-\cdots\right] \\
& +D_{j}\left(W^{\alpha}\right)\left[\frac{\partial \mathscr{L}}{\partial u_{i j}^{\alpha}}-D_{k}\left(\frac{\partial \mathscr{L}}{\partial u_{i j k}^{\alpha}}\right)+\cdots\right]+D_{j} D_{k}\left(W^{\alpha}\right)\left[\frac{\partial \mathscr{L}}{\partial u_{i j k}^{\alpha}}-\cdots\right],
\end{aligned}
$$

¿əe

$$
W^{\alpha}=\eta^{\alpha}-\xi^{j} u_{j}^{\alpha},
$$

а $\mathscr{L}$ - формальный лагранжиан (1.7). В формулах (1.21) формальный лагранжиан $\mathscr{L}$ следует писать в симметричной форме относительно всех смешанных производных $u_{i j}^{\alpha}, u_{i j k}^{\alpha}, \ldots . \quad$ В окончательных выражениях (1.21) для $C^{i}$ нужно исключить “лишние” зависимые переменные $v^{\alpha}$ вместе с производными, используя подстановку (1.12).

1.2.2. Законы сохранения, зависящие от времени, для уравнения Кадомцева-Петвиашвили. Воспользуемся уравнением Кадомцева-Петвиашвили (КП) [17]

$$
u_{t x}-u u_{x x}-u_{x}^{2}-u_{x x x x}=u_{y y}
$$

записанным в виде системы (см., например, [18] и содержащиеся там ссылки)

$$
u_{t}-u u_{x}-u_{x x x}-\omega_{y}=0, \quad \omega_{x}-u_{y}=0 .
$$


Нелинейная самосопряженность. Формальный лагранжиан (1.7) для уравнений (1.24) записывается в виде

$$
\mathscr{L}=v\left(u_{t}-u u_{x}-u_{x x x}-\omega_{y}\right)+z\left(\omega_{x}-u_{y}\right),
$$

а соотношение (1.6) дает следующую сопряженную систему для (1.24):

$$
v_{t}-u v_{x}-v_{x x x}-z_{y}=0, \quad z_{x}-v_{y}=0 .
$$

Уравнения (1.26) идентичны уравнениям КП (1.24) после подстановки

$$
v=u, \quad z=\omega
$$

Значит, система (1.24) является нелинейно самосопряженной.

Симметрии. Система (1.24) допускает бесконечномерную алгебру Ли, натянутую на операторы

$$
\begin{aligned}
& X_{f}=3 f \frac{\partial}{\partial t}+\left(f^{\prime} x+\frac{1}{2} f^{\prime \prime} y^{2}\right) \frac{\partial}{\partial x}+2 f^{\prime} y \frac{\partial}{\partial y} \\
&- {\left[2 f^{\prime} u+f^{\prime \prime} x+\frac{1}{2} f^{\prime \prime \prime} y^{2}\right] \frac{\partial}{\partial u} } \\
&- {\left[3 f^{\prime} \omega+f^{\prime \prime} y u+f^{\prime \prime \prime} x y+\frac{1}{6} f^{(4)} y^{3}\right] \frac{\partial}{\partial \omega}, } \\
& X_{g}=2 g \frac{\partial}{\partial y}+g^{\prime} y \frac{\partial}{\partial x}-g^{\prime \prime} y \frac{\partial}{\partial u}-\left[g^{\prime} u+g^{\prime \prime} x+\frac{1}{2} g^{\prime \prime \prime} y^{2}\right] \frac{\partial}{\partial \omega}, \\
& X_{h}=h \frac{\partial}{\partial x}-h^{\prime} \frac{\partial}{\partial u}-h^{\prime \prime} y \frac{\partial}{\partial \omega},
\end{aligned}
$$

где $f, g, h$ - три произвольные функции от $t$. Мы не будем использовать очевидную симметрию

$$
X_{\alpha}=\alpha(t) \frac{\partial}{\partial \omega}
$$

описывающую прибавление к $\omega$ произвольной функции от $t$.

Операторы (1.28)-(1.30), записанные без членов с $\partial / \partial \omega$, образуют бесконечномерную алгебру Ли симметрий уравнения КП (1.23).

Законы сохранения. Теорема Нётер не применима к системе (1.24), в то время как теорема 1.1 применима. Подставляя в формулу (1.21) формальный лагранжиан (1.25) и симметрии (1.28), а затем исключая $v, z$ с помощью подстановки (1.27), мы приходим к закону сохранения

$$
\left[D_{t}\left(C^{1}\right)+D_{x}\left(C^{2}\right)+D_{y}\left(C^{3}\right)\right]_{(1.24)}=0
$$


со следующими компонентами сохраняющегося вектора $C=\left(C^{1}, C^{2}, C^{3}\right)$ :

$$
\begin{aligned}
C^{1}= & -\frac{1}{2} f^{\prime} u^{2}-\left(x f^{\prime \prime}+\frac{1}{2} y^{2} f^{\prime \prime \prime}\right) u, \\
C^{2}= & \left(u u_{x x}+\frac{1}{3} u^{3}-\frac{1}{2} u_{x}^{2}-\frac{1}{2} \omega^{2}\right) f^{\prime}+\left(x u_{x x}+\frac{1}{2} x u^{2}-u_{x}\right) f^{\prime \prime} \\
& \quad+\frac{1}{4}\left(y^{2} u^{2}+2 y^{2} u_{x x}-4 x y \omega\right) f^{\prime \prime \prime}-\frac{1}{6} y^{3} \omega f^{(4)}, \\
C^{3}= & u \omega f^{\prime}+x \omega f^{\prime \prime}+\left(x y u+\frac{1}{2} y^{2} \omega\right) f^{\prime \prime \prime}+\frac{1}{6} y^{3} u f^{(4)} .
\end{aligned}
$$

Закон сохранения (1.31) для вектора (1.32) выполняется в виде

$$
\begin{aligned}
D_{t}\left(C^{1}\right)+D_{x}\left(C^{2}\right)+D_{y}\left(C^{3}\right)=( & \left.u f^{\prime}+x f^{\prime \prime}+\frac{1}{2} y^{2} f^{\prime \prime \prime}\right)\left(u_{x x x}+u u_{x}+\omega_{y}-u_{t}\right) \\
& +\left(\omega f^{\prime}+x y f^{\prime \prime \prime}+\frac{1}{6} y^{3} f^{(4)}\right)\left(u_{y}-\omega_{x}\right) .
\end{aligned}
$$

Так как $f=f(t)$ - произвольная функция, то формула (1.32) дает бесконечное множество сохраняющихся векторов. Напомним, что нижний индекс (1.24) в уравнении (1.31) указывает, что выражение с этим индексом рассматривается на множестве решений уравнений (1.24).

Симметрии (1.29), (1.30) также дают нетривиальные законы сохранения, которые легко вычислить по формуле (1.21).

Теорема Нётер может быть применена к уравнению КП, если записать его в терминах потенциала $\phi$, определяемого уравнениями

$$
u=\phi_{x}, \quad \omega=\phi_{y} .
$$

При этом второе уравнение системы (1.24) гарантирует совместность приведенной выше переопределенной системы для функции $\phi$, а первое уравнение системы (1.24) дает следующее потенциальное уравнение КП:

$$
\phi_{x t}-\phi_{x} \phi_{x x}-\phi_{x x x x}-\phi_{y y}=0 \text {. }
$$

Это уравнение имеет лагранжиан

$$
L=-\frac{1}{2} \phi_{x} \phi_{t}+\frac{1}{6} \phi_{x}^{3}+\frac{1}{2} \phi_{y}^{2}-\frac{1}{2} \phi_{x x}^{2}
$$

Теперь можно применить теорему Нётер. Более детальное обсуждение такого подхода имеется в статье [6] и приведенной там библиографии.

1.2.3. Модель распространения ультракоротких световых импульсов в нелинейных средах. Здесь речь пойдет о следующем нелинейном дифференциальном уравнении:

$$
u_{x t}=u+\frac{1}{2} u^{2} u_{x x}+u u_{x}^{2}
$$


Оно было предложено в работе [19] в качестве математической модели для описания распространения ультракоротких световых импульсов в нелинейных средах, например, в стекловолокне. Вывод этой модели основан на рассмотрении распространения линейно поляризованного света. При этом предполагается, что свет распространяется в инфракрасной области. Последний шаг построения модели основан на методе многих масштабов.

В работах [20], [21] показано, что уравнение (1.34) связано с уравнением $u_{x y}=\sin u$ неточечным преобразованием, получаемым как цепочка дифференциальных подстановок. Используя эту связь, построено решение типа уединенной волны, а также найдены пара Лакса и оператор рекурсии.

Уравнение (1.34) не имеет вида закона сохранения. Этот вид был найден в препринте [5] методом нелинейной самосопряженности. Приведем здесь этот результат. Формальный лагранжиан уравнения (1.34) имеет вид

$$
\mathscr{L}=v\left[u_{x t}-u-\frac{1}{2} u^{2} u_{x x}-u u_{x}^{2}\right]
$$

и приводит к следующему сопряженному уравнению для (1.34):

$$
v_{x t}=v+\frac{1}{2} u^{2} v_{x x}
$$

ПреДЛОЖЕНИЕ 1.4. Уравнение (1.34) является нелинейно самосопряженнъм, причем решение сопряженного уравнения дается дифберенциальной подстановкой (1.12) следующего вида:

$$
v=u_{t}-\frac{1}{2} u^{2} u_{x}
$$

Максимальная алгебра Ли точечных симметрий уравнения (1.34) трехмерна и натянута на следующие операторы:

$$
X_{1}=\frac{\partial}{\partial t}, \quad X_{2}=\frac{\partial}{\partial x}, \quad X_{3}=u \frac{\partial}{\partial u}+x \frac{\partial}{\partial x}-t \frac{\partial}{\partial t} .
$$

Применение метода нелинейной самосопряженности к операторам (1.38) показывает, что только оператор $X_{3}$ дает нетривиальный закон сохранения

$$
D_{t}\left(C^{1}\right)+D_{x}\left(C^{2}\right)=0
$$

а именно сохраняющийся вектор

$$
\begin{aligned}
& C^{1}=u^{2}, \\
& C^{2}=u^{2} u_{x} u_{t}-u_{t}^{2}-\frac{1}{4} u^{4}-\frac{1}{4} u^{4} u_{x}^{2} .
\end{aligned}
$$

Закон сохранения (1.39) для этого вектора выполняется в виде

$$
D_{t}\left(C^{1}\right)+D_{x}\left(C^{2}\right)=2\left(u_{t}-\frac{1}{2} u^{2} u_{x}\right)\left(u+\frac{1}{2} u^{2} u_{x x}+u u_{x}^{2}-u_{x t}\right) .
$$


ПрЕДЛОжЕНИЕ 1.5. Точечные симметрии (1.38) уравнения (1.34) дают один нетривиальный сохраняющийся вектор (1.40). Соответственно, уравнение (1.34) может быть записано в виде следующего закона сохранения:

$$
D_{t}\left(u^{2}\right)+D_{x}\left(u^{2} u_{x} u_{t}-u_{t}^{2}-\frac{1}{4} u^{4}-\frac{1}{4} u^{4} u_{x}^{2}\right)=0 .
$$

\section{Глава 2. Построение решений методом законов сохранения}

В этой главе описывается новый метод, метод законов сохранения, позволяющий строить точные решения систем нелинейных уравнений в частных производных. С помощью этого метода находятся новые точные решения, отличные от инвариантно-групповых решений, для газа Чаплыгина [7], произвольного одномерного политропного движения газа [10] и нелинейной диффузии в анизотропных средах с источником [9].

\section{1. Метод законов сохранения}

Несмотря на всеобщее признание важности законов сохранения как полезного инструмента при составлении и изучении математических моделей, численном решении дифференциальных уравнений и т. д., часто задается вопрос, могут ли законы сохранения быть полезны при аналитическом решении систем дифференциальных уравнений в частных производных.

Мы показываем, что ответ на этот вопрос положительный, и иллюстрируем это положение несколькими примерами, взятыми из нелинейных математических моделей, имеющих практическое значение.

В данном разделе обсуждается разница между законами сохранения для обыкновенных дифференциальных уравнений и дифференциальных уравнений в частных производных с точки зрения их использования при решении дифференциальных уравнений.

2.1.1. Обыкновенные дифференциальные уравнения. В случае обыкновенных дифференциальных уравнений

$$
F\left(x, y, y^{\prime}, \ldots, y^{(s)}\right)=0
$$

любой нетривиальный закон сохранения

$$
\left[\left(D_{x} \psi\left(x, y, y^{\prime}, \ldots, y^{(s-1)}\right)\right)\right]_{(2.1)}=0
$$

дает первый интеграл

$$
\psi\left(x, y, y^{\prime}, \ldots, y^{(s-1)}\right)=C, \quad C=\text { const } .
$$

Таким образом, знание законов сохранения для системы обыкновенных дифференциальных уравнений позволяет понизить порядок системы или найти ее общее решение, если известно достаточное количество законов сохранения. 
Часто бывает так, что важные физические свойства задачи, описываемой обыкновенными дифференциальными уравнениями, можно выразить напрямую в терминах законов сохранения, не решая эти дифференциальные уравнения. Рассмотрим, например, задачу двух тел (планета и солнце), описываемую уравнением Ньютона

$$
m \mathbf{x}^{\prime \prime}=\alpha \frac{\mathbf{x}}{r^{3}}
$$

для вектора $\mathbf{x}$ положения планеты. Здесь $r=|\mathbf{x}|-$ расстояние от планеты до солнца, $\mathbf{v}=\mathbf{x}^{\prime}-$ скорость движения планеты, $\mathbf{x}^{\prime \prime}-$ производная второго порядка от вектора $\mathbf{x}$ по времени, а $\alpha$ - константа, пропорциональная массе $m$ планеты. Уравнение (2.4) обладает, в частности, законом сохранения вектора момента количества движения

$$
\mathbf{M}=m(\mathbf{x} \times \mathbf{v})
$$

и вектора Лапласа

$$
\mathbf{A}=\mathbf{v} \times \mathbf{M}+\alpha \frac{\mathbf{x}}{r}
$$

Уже в 1798 г. Лаплас показал, что второй и первый законы Кеплера можно вывести непосредственно из сохранения векторов (2.5) и (2.6) соответственно, не решая систему обыкновенных дифференциальных уравнений второго порядка (2.4).

ЗАмЕчАниЕ 2.1. Широко известно, что сохранение момента количества движения (2.5) является следствием инвариантности уравнения Ньютона (2.4) относительно группы вращений по пространственным переменным х. Таким образом, второй закон Кеплера следует из вращательной симметрии уравнений Ньютона (2.4). Вопрос же о наличии специальной симметрии уравнения Ньютона, приводящего к вектору Лапласа (2.6) или, иначе говоря, к первому закону Кеплера, долгое время оставался открытым. Эта симметрия была найдена только в 1983 г. Н. Х. Ибрагимовым (см. [12; § 25]). Оказалось, что эта симметрия существенно более сложная, чем вращательная симметрия, а именно, она выражается в терминах касательных преобразований бесконечного порядка (группа Ли-Бэклунда).

2.1.2. Системы уравнений в частных производных. В случае уравнений в частных производных ситуация выглядит иначе. А именно, знание закона сохранения (1.1) не позволяет, вообще говоря, понизить порядок системы (1.4) и не ведет к какому-либо решению этой системы. В работе [7] был предложен простой метод построения решений систем уравнений в частных производных с помощью законов сохранения.

Суть этого нового метода состоит в следующем. Пусть вектор (1.3) с компонентами (1.2) является сохраняющимся для системы (1.4). Найдем частные решения системы (1.4) путем добавления к ней следующих дифференциальных 
уравнений:

$$
\begin{aligned}
& D_{1}\left[C^{1}\left(x, u, u_{(1)}, \ldots\right)\right]=0, \\
& D_{2}\left[C^{2}\left(x, u, u_{(1)}, \ldots\right)\right]=0, \\
& \ldots \ldots \ldots \ldots \ldots \ldots \ldots \\
& D_{n}\left[C^{n}\left(x, u, u_{(1)}, \ldots\right)\right]=0 .
\end{aligned}
$$

Дополнительные дифференциальные уравнения (2.7) будут называться дифференциальными связями.

Заметим, что переопределенная система $m+n$ уравнений $(1.4),(2.7)$ содержит лишь $m+n-1$ независимых уравнений в силу закона сохранения (1.1). $\mathrm{K}$ ним нужно применить известную технику исследования условий совместности переопределенных систем. Можно ожидать, что в данном случае задача будет несколько проще, чем в общей ситуации, так как дифференциальные связи (2.7) взяты не произвольно, а из законов сохранения системы (1.4), и потому выражают некоторые внутренние свойства множества решений этой системы. Однако обсуждение этой задачи выходит за рамки данного обзора.

\section{2. Применение к газу Чаплыгина}

Газ Чаплыгина описывается уравнениями политропной одномерной газовой динамики с показателем политропы $\gamma=-1$ :

$$
\begin{aligned}
& v_{t}+v v_{x}+\frac{1}{\rho} p_{x}=0, \\
& \rho_{t}+v \rho_{x}+\rho v_{x}=0 \\
& p_{t}+v p_{x}-p v_{x}=0 .
\end{aligned}
$$

2.2.1. Локальные и нелокальные симметрии. Уравнения (2.8) имеют шестимерную алгебру Ли точечных симметрий с базисом

$$
\begin{gathered}
X_{1}=\frac{\partial}{\partial t}, \quad X_{2}=\frac{\partial}{\partial x}, \quad X_{3}=t \frac{\partial}{\partial x}+\frac{\partial}{\partial v}, \quad X_{4}=t \frac{\partial}{\partial t}+x \frac{\partial}{\partial x}, \\
X_{5}=\rho \frac{\partial}{\partial \rho}+p \frac{\partial}{\partial p}, \quad X_{6}=t \frac{\partial}{\partial t}-v \frac{\partial}{\partial v}+2 \rho \frac{\partial}{\partial \rho} .
\end{gathered}
$$

Кроме того, газ Чаплыгина имеет две нелокальные симметрии [22]

$$
\begin{aligned}
& X_{7}=\sigma \frac{\partial}{\partial x}-\frac{\partial}{\partial p}+\frac{\rho}{p} \frac{\partial}{\partial \rho} \\
& X_{8}=\left(\frac{t^{2}}{2}+s\right) \frac{\partial}{\partial x}+t \frac{\partial}{\partial v}-\tau \frac{\partial}{\partial p}+\frac{\rho \tau}{p} \frac{\partial}{\partial \rho} .
\end{aligned}
$$

Здесь $\tau, s, \sigma$ представляют собой следующие нелокалъные переменные:

$$
\tau=\int \rho d x, \quad s=-\int \frac{\tau}{p} d x, \quad \sigma=-\int \frac{d x}{p},
$$


которые могут быть также заданы совместными переопределенными системами уравнений

$$
\begin{aligned}
\tau_{x} & =\rho, & \tau_{t} & =-v \rho, \\
s_{x} & =-\frac{\tau}{p}, & s_{t} & =\frac{v \tau}{p}, \\
\sigma_{x} & =-\frac{1}{p}, & \sigma_{t} & =\frac{v}{p} .
\end{aligned}
$$

Операторы растяжений $X_{4}, X_{5}, X_{6}$ надо продолжить на нелокальные переменные. Это продолжение имеет вид

$$
\begin{aligned}
& X_{4}^{\prime}=t \frac{\partial}{\partial t}+x \frac{\partial}{\partial x}+\tau \frac{\partial}{\partial \tau}+2 s \frac{\partial}{\partial s}+\sigma \frac{\partial}{\partial \sigma}, \\
& X_{5}^{\prime}=\rho \frac{\partial}{\partial \rho}+p \frac{\partial}{\partial p}+\tau \frac{\partial}{\partial \tau}-\sigma \frac{\partial}{\partial \sigma} \\
& X_{6}^{\prime}=t \frac{\partial}{\partial t}-v \frac{\partial}{\partial v}+2 \rho \frac{\partial}{\partial \rho}+2 \tau \frac{\partial}{\partial \tau}+2 s \frac{\partial}{\partial s} .
\end{aligned}
$$

Операторы (2.10) и (2.13) вместе с операторами $X_{1}, X_{2}, X_{3}$ из (2.9) образуют восьмимерную алгебру Ли $L_{8}$ с таблицей коммутаторов

\begin{tabular}{||c||c|c|c|c|c|c|c|c||}
\hline & $X_{1}$ & $X_{2}$ & $X_{3}$ & $X_{4}^{\prime}$ & $X_{5}^{\prime}$ & $X_{6}^{\prime}$ & $X_{7}$ & $X_{8}$ \\
\hline \hline$X_{1}$ & 0 & 0 & $X_{2}$ & $X_{1}$ & 0 & $X_{1}$ & 0 & $X_{3}$ \\
\hline$X_{2}$ & 0 & 0 & 0 & $X_{2}$ & 0 & 0 & 0 & 0 \\
\hline$X_{3}$ & $-X_{2}$ & 0 & 0 & 0 & 0 & $-X_{3}$ & 0 & 0 \\
\hline$X_{4}^{\prime}$ & $-X_{1}$ & $-X_{2}$ & 0 & 0 & 0 & 0 & 0 & $X_{8}$ \\
\hline$X_{5}^{\prime}$ & 0 & 0 & 0 & 0 & 0 & 0 & $-X_{7}$ & 0 \\
\hline$X_{6}^{\prime}$ & $-X_{1}$ & 0 & $X_{3}$ & 0 & 0 & 0 & 0 & $2 X_{8}$ \\
\hline$X_{7}$ & 0 & 0 & 0 & 0 & $X_{7}$ & 0 & 0 & 0 \\
\hline$X_{8}$ & $-X_{3}$ & 0 & 0 & $-X_{8}$ & 0 & $-2 X_{8}$ & 0 & 0 \\
\hline
\end{tabular}

2.2.2. Локальные законы сохранения. Локальные законы сохранения для газа Чаплыгина, т. е. те законы, которые включают физические переменные $t, x, v, \rho, p$, являются выражением классических утверждений о сохранении массы, энергии, количества движения и центра масс. В дифференциальной форме (1.1) они записываются в виде следующих уравнений:

$$
\begin{aligned}
& D_{t}(\rho)+D_{x}(\rho v)=\rho_{t}+v \rho_{x}+\rho v_{x}, \\
& D_{t}\left(\rho v^{2}-p\right)+D_{x}\left(p v+\rho v^{3}\right)=2 \rho v\left(v_{t}+v v_{x}+\frac{1}{\rho} p_{x}\right) \\
& +v^{2}\left(\rho_{t}+v \rho_{x}+\rho v_{x}\right)-\left(p_{t}+v p_{x}-p v_{x}\right), \\
& D_{t}(\rho v)+D_{x}\left(p+\rho v^{2}\right)=\rho\left(v_{t}+v v_{x}+\frac{1}{\rho} p_{x}\right)+v\left(\rho_{t}+v \rho_{x}+\rho v_{x}\right), \\
& D_{t}(t \rho v-x \rho)+D_{x}\left(t p+t \rho v^{2}-x \rho v\right) \\
& =t \rho\left(v_{t}+v v_{x}+\frac{1}{\rho} p_{x}\right)+(t v-x)\left(\rho_{t}+v \rho_{x}+\rho v_{x}\right) .
\end{aligned}
$$


2.2.3. Нелокальные законы сохранения. Газ Чаплыгина, в отличие от общего политропного газа, имеет нелокальные законы сохранения. Они получаются подстановкой нелокальных симметрий (2.10) в формулу (1.21). При этом используется предложение 1.2. Применив его к локальным законам сохранения (2.14)-(2.17), приходим к следующему результату [5].

ПРЕДЛОЖЕНИЕ 2.1. Нелокалънъе симметрии (2.10) газа Чапльгина дают четыре нелокальных закона сохранения

$$
\left[D_{t}\left(C^{1}\right)+D_{x}\left(C^{2}\right)\right]_{(2.8)}=0
$$

с сохраняющимися векторами $C=\left(C^{1}, C^{2}\right)$, имеющими следующие коордиHamb:

$$
\begin{array}{ll}
C^{1}=\sigma \rho, & C^{2}=\sigma \rho v, \\
C^{1}=t \rho v+\tau, & C^{2}=t\left(\rho v^{2}+p\right), \\
C^{1}=t \rho, & C^{2}=t \rho v-\tau, \\
C^{1}=\left(t^{2}-2 s\right) \rho, & C^{2}=\left(t^{2}-2 s\right) \rho v-2 t \tau .
\end{array}
$$

Можно показать, что закон сохранения (2.18) для векторов (2.19)-(2.22) имеет вид

$$
\begin{aligned}
& D_{t}(\sigma \rho)+D_{x}(\sigma \rho v)=\sigma\left(\rho_{t}+v \rho_{x}+\rho v_{x}\right) \\
& D_{t}(t \rho v+\tau)+D_{x}\left(t\left(\rho v^{2}+p\right)\right) \\
& \quad=t \rho\left(v_{t}+v v_{x}+\frac{1}{\rho} p_{x}\right)+t v\left(\rho_{t}+v \rho_{x}+\rho v_{x}\right) \\
& D_{t}(t \rho)+D_{x}(t \rho v-\tau)=t\left(\rho_{t}+v \rho_{x}+\rho v_{x}\right) \\
& \quad D_{t}\left(\left(t^{2}-2 s\right) \rho\right)+D_{x}\left(\left(t^{2}-2 s\right) \rho v-2 t \tau\right) \\
& \quad=\left(t^{2}-2 s\right)\left(\rho_{t}+v \rho_{x}+\rho v_{x}\right)
\end{aligned}
$$

соответственно.

2.2.4. Решения, полученные из закона сохранения массы. Здесь мы используем закон сохранения (2.14) с сохраняющимся вектором

$$
C^{1}=\rho, \quad C^{2}=\rho v .
$$

В этом случае дифференциальные связи (2.7) имеют вид

$$
D_{t}(\rho)=0, \quad D_{x}(\rho v)=0
$$

или, в проинтегрированной форме,

$$
\rho=g(x), \quad \rho v=h(t) .
$$


Итак, мы ищем решения вида

$$
\rho=g(x), \quad v=\frac{h(t)}{g(x)} .
$$

Функции (2.27) удовлетворяют второму уравнению системы (2.8) благодаря закону сохранения (2.14). Поэтому остается подставить (2.27) в первое и третье уравнения из (2.8). Получившиеся в результате этой подстановки уравнения можно разрешить относительно производных $p$ :

$$
\begin{aligned}
& p_{x}=-h^{\prime}+\frac{h^{2} g^{\prime}}{g^{2}} \\
& p_{t}=-\frac{h g^{\prime}}{g^{2}} p+\frac{h h^{\prime}}{g}-\frac{h^{3} g^{\prime}}{g^{3}} .
\end{aligned}
$$

Условие совместности $p_{x t}=p_{t x}$ системы $(2.28)$ приводит к уравнению

$$
\left(g^{\prime \prime}-2 \frac{g^{\prime 2}}{g}\right) p=g^{2} \frac{h^{\prime \prime}}{h}-2 g^{\prime} h^{\prime}-h^{2} \frac{g^{\prime \prime}}{g}+2 h^{2} \frac{g^{2}}{g^{2}} .
$$

Продолжая процедуру выписывания условий совместности переопределенной системы (2.28)-(2.29) для $p$, можно получить систему дифференциальных уравнений для нахождения функций $g(x)$ и $h(t)$. Однако вычисления значительно сократятся, если рассмотреть частный случай, когда коэффициент перед $p$ в уравнении (2.29) обращается в нуль:

$$
g^{\prime \prime}-2 \frac{g^{\prime 2}}{g}=0
$$

Решение уравнения (2.30) имеет вид

$$
g(x)=\frac{1}{a x+b}, \quad a, b=\text { const } .
$$

Подставив (2.31) в (2.29), получим

$$
h^{\prime \prime}+2 a h h^{\prime}=0
$$

Отсюда следует, что

$$
h(t)=k \tan (c-a k t)
$$

если $a \neq 0$, и

$$
h(t)=A t+B
$$

если $a=0$. Чтобы исключить тривиальное решение с постоянными $v, \rho, p$, мы будем предполагать, что $A \neq 0$. 
Если постоянная $a$ в (2.31) отлична от нуля, мы подставляем (2.31) и (2.33) в уравнения (2.28), интегрируем их и получаем

$$
p=k^{2}(a x+b)+Q \cos (c-a k t), \quad Q=\text { const } .
$$

В случае $a=0$ аналогичные вычисления дают

$$
p=-A x+\frac{b}{2} A^{2} t^{2}+A B b t+Q, \quad Q=\text { const } .
$$

Итак, с помощью закона сохранения массы мы получили точные решения

$$
\begin{aligned}
\rho & =\frac{1}{a x+b}, \quad a \neq 0, \\
v & =k(a x+b) \tan (c-a k t), \\
p & =k^{2}(a x+b)+Q \cos (c-a k t)
\end{aligned}
$$

и

$$
\begin{aligned}
\rho & =\frac{1}{b}, \quad b \neq 0, \\
v & =b(A t+B), \\
p & =-A x+\frac{b}{2} A^{2} t^{2}+A B b t+Q .
\end{aligned}
$$

Возникает вопрос, нельзя ли получить решения (2.37) и (2.38) как инвариантно-групповые решения с помощью некоторых подалгебр алгебры Ли точечных симметрий (2.9). Для этого нужно изучить вопрос, существуют ли постоянные $l_{1}, \ldots, l_{6}$, которые не все равны нулю одновременно и для которых оператор

$$
\begin{aligned}
& X=l_{1} X_{1}+\cdots+l_{6} X_{6}=\left[l_{1}+\left(l_{4}+l_{6}\right) t\right] \frac{\partial}{\partial t}+\left[l_{2}+l_{3} t+l_{4} x\right] \frac{\partial}{\partial x} \\
&+\left[l_{3}-l_{6} v\right] \frac{\partial}{\partial v}+\left[l_{5}+2 l_{6}\right] \rho \frac{\partial}{\partial \rho}+l_{5} p \frac{\partial}{\partial p}
\end{aligned}
$$

удовлетворяет условиям инвариантности

$$
\begin{aligned}
& \text { (i) }\left[X\left(\rho-\frac{1}{a x+b}\right)\right]_{(2.37)}=0, \\
& \text { (ii) }[X(v-k(a x+b) \tan (c-a k t))]_{(2.37)}=0, \\
& \text { (iii) }\left[X\left(p-k^{2}(a x+b)-Q \cos (c-a k t)\right)\right]_{(2.37)}=0
\end{aligned}
$$

И

$$
\begin{aligned}
& \text { (i) }\left[X\left(\rho-\frac{1}{b}\right)\right]_{(2.38)}=0 \\
& \text { (ii) }[X(v-b(A t+B))]_{(2.38)}=0 \\
& \text { (iii) }\left[X\left(p+A x-\frac{b}{2} A^{2} t^{2}-A B b t-Q\right)\right]_{(2.38)}=0
\end{aligned}
$$

для решений (2.37) и (2.38) соответственно. 
ПреДлОЖЕНИЕ 2.2. Решение (2.37) не является инвариантным решением.

ДокАзАтельство. Подставляя выражение (2.39) для $X$ в уравнение (i) системы $(2.40)$ и умножая на $(a x+b)^{2}$, получаем

$$
\left(l_{5}+2 l_{6}\right)(a x+b)+a\left(l_{2}+l_{3} t+l_{4} x\right)=0 .
$$

Отсюда, путем расщепления по $t, x$, т. е. приравнивая к нулю члены с $t, x$ и остальные члены, мы получаем следующие три уравнения:

$$
\begin{aligned}
l_{3} & =0, \\
l_{5}+2 l_{6}+l_{4} & =0, \\
a l_{2}+b\left(l_{5}+2 l_{6}\right) & =0 .
\end{aligned}
$$

Поступая аналогично с уравнением (ii) системы (2.40), принимая во внимание уравнение $(2.42)$ и умножая на $\cos (c-a k t)$, получаем

$$
\frac{a k(a x+b)\left[l_{1}+\left(l_{4}+l_{6}\right) t\right]}{\cos (c-a k t)}-\left[l_{6}(a x+b)+a\left(l_{2}+l_{4} x\right)\right] \sin (c-a k t)=0 .
$$

Отсюда, приравнивая к нулю члены с различными тригонометрическими функциями и расщепляя по $t, x$ как выше, приходим к следующим уравнениям:

$$
\begin{aligned}
l_{1} & =0, \\
l_{4}+l_{6} & =0, \\
a l_{2}+b l_{6} & =0 .
\end{aligned}
$$

Заметим, что уравнения (2.43) и (2.46) дают

$$
l_{5}+l_{6}=0
$$

и что, благодаря последнему уравнению, два уравнения (2.44) и (2.47) совпадают. Следовательно, уравнения (2.42)-(2.47) сводятся к следующим:

$$
l_{1}=l_{3}=0, \quad l_{4}+l_{6}=0, \quad l_{5}+l_{6}=0, \quad a l_{2}+b l_{6}=0 .
$$

Наконец, мы записываем уравнение (iii) системы (2.40), принимая в расчет уравнения (2.48), и получаем

$$
l_{5}\left[k^{2}(a x+b)+Q \cos (c-a k t)\right]-a k^{2}\left(l_{2}+l_{4} x\right)=0,
$$

откуда следует, что

$$
l_{2}=l_{4}=l_{5}=0 .
$$

Теперь второе уравнение в $(2.48)$ дает $l_{6}=0$, и уравнения $(2.48),(2.49)$ принимают вид

$$
l_{1}=l_{2}=l_{3}=l_{4}=l_{4}=l_{6}=0 .
$$

Таким образом, оператор (2.39) обращается в нуль. Это доказывает наше утверждение. 
ПреДлОЖЕНИЕ 2.3. Решение (2.38) не является инвариантным решением. ДокАзАтЕльство. Уравнение (i) в (2.41) имеет вид

$$
l_{5}+2 l_{6}=0 .
$$

Уравнение (ii) в (2.41) записывается в виде

$$
l_{3}-b(A t+B) l_{6}-b A\left[l_{1}+\left(l_{4}+l_{6}\right) t\right]=0
$$

и после расщепления по $t$ дает

$$
\begin{aligned}
l_{4}+2 l_{6} & =0, \\
l_{3}-b\left(A l_{1}+B l_{6}\right) & =0 .
\end{aligned}
$$

Уравнение (iii) в (2.41) имеет вид

$$
\begin{gathered}
\left(-A x+\frac{b}{2} A^{2} t^{2}+A B b t+Q\right) l_{5}+A\left(l_{2}+l_{3} t+l_{4} x\right) \\
+A b(A t-B)\left[l_{1}+\left(l_{4}+l_{6}\right) t\right]=0 .
\end{gathered}
$$

Приравнивая к нулю коэффициент при $t^{2}$ в $(2.53)$, получаем

$$
l_{5}+2 l_{6}+2 l_{4}=0
$$

откуда, в силу уравнения (2.50), следует, что $l_{4}=0$. Это уравнение вместе с уравнениями (2.50), (2.51) дает

$$
l_{4}=l_{5}=l_{6}=0 .
$$

Уравнение (2.52) теперь записывается в виде

$$
l_{3}-b A l_{1}=0 .
$$

В силу уравнений (2.54), уравнение (2.53) принимает вид

$$
A\left(l_{2}+l_{3} t\right)+A b(A t-B) l_{1}=0,
$$

откуда

$$
l_{3}+b A l_{1}=0, \quad l_{2}-b B l_{1}=0 .
$$

Первое уравнение в (2.56) вместе с уравнением (2.55) показывают, что

$$
l_{1}=l_{3}=0,
$$

а второе уравнение в $(2.56)$ дает $l_{2}=0$. Итак, мы получаем уравнения

$$
l_{1}=l_{2}=l_{3}=l_{4}=l_{5}=l_{6}=0,
$$

что доказывает наше утверждение.

ЗАмЕчАниЕ 2.2. Из предложений 2.2, 2.3 следует также, что решения (2.37), (2.38) не могут быть и частично инвариантными решениями. Действительно, как показал Л. В. Овсянников [3], все частично инвариантные решения уравнений произвольного политропного одномерного движения газа являются инвариантными решениями. 
2.2.5. Решения, полученные из закона сохранения количества движения. Законы сохранения (2.15)-(2.17) энергии, количества движения и центра масс дают уравнения вида

$$
\begin{aligned}
\rho v^{2}-p & =g(x), & p v+\rho v^{3} & =h(t), \\
\rho v & =g(x), & p+\rho v^{2} & =h(t), \\
t \rho v-x \rho & =g(x), & t p+t \rho v^{2}-x \rho v & =h(t) .
\end{aligned}
$$

Нелокальные сохраняющиеся векторы (2.19)-(2.22) приводят к уравнениям вида

$$
\begin{aligned}
\sigma \rho & =g(x), & \sigma \rho v & =h(t), \\
t \rho v+\tau & =g(x), & p+\rho v^{2} & =h(t), \\
t \rho & =g(x), & t \rho v-\tau & =h(t), \\
\left(t^{2}-2 s\right) \rho & =g(x), & \left(t^{2}-2 s\right) \rho v-2 t \tau & =h(t) .
\end{aligned}
$$

Здесь мы применим метод закона сохранения к сохранению количества движения, т. е. используем уравнения $\left(2.16^{\prime}\right)$. Итак, мы ищем решения вида

$$
\rho=\frac{g(x)}{v}, \quad p=h(t)-g(x) v .
$$

Подстановка выражений (2.57) в первое уравнение системы (2.8) дает

$$
v_{t}-\frac{g^{\prime}(x)}{g(x)} v=0 .
$$

Благодаря уравнениям (2.57), (2.58), третье уравнение системы (2.8) принимает вид

$$
h^{\prime}(t)-2 g^{\prime}(x) v^{2}-h(t) v_{x}=0,
$$

а второе уравнение системы (2.8) выполняется тождественно.

Проинтегрировав уравнение (2.58) по $t$, получаем его общее решение

$$
v=\frac{g(x)}{F(x)-t g^{\prime}(x)}
$$

с произвольной функцией $F(x)$. Можно было бы подставить выражение $(2.60)$ для $v$ в (2.59) и изучить полученное уравнение для двух неизвестных функций $g(x)$ и $h(t)$. Но мы упростим вычисления, положив $F(x)=0$. Тогда

$$
v=-\frac{g(x)}{t g^{\prime}(x)}
$$

и уравнение (2.59) принимает вид

$$
h^{\prime}(t)-2 \frac{g^{2}(x)}{g^{\prime}(x)} \frac{1}{t^{2}}+\frac{h(t)}{t}-\frac{g(x) g^{\prime \prime}(x)}{g^{\prime 2}(x)} \frac{h(t)}{t}=0 .
$$


Для разделения переменных положим

$$
h(t)=\frac{\alpha}{t}
$$

с произвольной постоянной $\alpha \neq 0$ и приведем уравнение $(2.62)$ к виду

$$
\alpha g^{\prime \prime}+2 g g^{\prime}=0
$$

или

$$
\left(\alpha g^{\prime}\right)^{\prime}+\left(g^{2}\right)^{\prime}=0
$$

Отсюда

$$
\alpha g^{\prime}+g^{2}=K, \quad K=\text { const } .
$$

Решение уравнения (2.64) зависит от знака постоянной $K$. Пусть $K=$ $\beta^{2}>0$. Тогда уравнение (2.64) записывается в виде

$$
\frac{d g}{g^{2}-\beta^{2}}=-\frac{1}{\alpha} d x
$$

и интегрирование дает

$$
g=\frac{\alpha \sigma}{2} \frac{1+C \mathrm{e}^{-\sigma x}}{1-C \mathrm{e}^{-\sigma x}},
$$

где $C$ - произвольная постоянная, а $\sigma=2 \beta / \alpha$.

Если $K=-\mu^{2}<0$, то решение уравнения (2.64) задается формулой

$$
g=\alpha \omega \tan (C-\omega x)
$$

где $C$ - произвольная постоянная, а $\omega=\mu / \alpha$.

Уравнения (2.65), (2.61), (2.57) и (2.63) дают следующее решение системы (2.8):

$$
\begin{aligned}
& v=\frac{1}{2 C \sigma t}\left(1-C^{2} \mathrm{e}^{-2 \sigma x}\right) \mathrm{e}^{\sigma x}, \\
& \rho=C \frac{\alpha \sigma^{2} t \mathrm{e}^{-\sigma x}}{\left(1-C \mathrm{e}^{-\sigma x}\right)^{2}}, \\
& p=\frac{\alpha}{t}\left[1-\frac{1}{4 C}\left(1+C \mathrm{e}^{-\sigma x}\right)^{2} \mathrm{e}^{\sigma x}\right] .
\end{aligned}
$$

Уравнения (2.66), (2.61), (2.57) и (2.63) дают следующее решение системы (2.8):

$$
\begin{aligned}
v & =\frac{1}{\omega t} \sin (C-\omega x) \cos (C-\omega x), \\
\rho & =\frac{\alpha \omega^{2} t}{\cos ^{2}(C-\omega x)}, \\
p & =\frac{\alpha}{t} \cos ^{2}(C-\omega x) .
\end{aligned}
$$


Повторяя процедуру, изложенную в доказательстве предложений 2.2, 2.3, и используя замечание 2.2, можно показать, что решения (2.67) и (2.68) не являются инвариантными или частично инвариантными решениями относительно некоторых подалгебр алгебры Ли точечных симметрий (2.9).

\section{3. Одномерный политропный газ}

Точные решения для уравнений произвольного политропного газа

$$
\begin{gathered}
v_{t}+v v_{x}+\frac{1}{\rho} p_{x}=0, \\
\rho_{t}+v \rho_{x}+\rho v_{x}=0, \\
p_{t}+v p_{x}+\gamma p v_{x}=0
\end{gathered}
$$

могут быть построены методом законов сохранения по тому же алгоритму, который применялся в случае газа Чаплыгина. Конкретные вычисления, однако, являются более сложными и изложены в работе [10]. Здесь мы приводим два новых точных решения, которые отличны от инвариантно-групповых решений. Эти решения основаны на законе сохранения массы

$$
\rho_{t}+v \rho_{x}+\rho v_{x} \equiv D_{t}(\rho)+D_{x}(\rho v)=0 .
$$

Метод законов сохранения показывает, что решения надо искать в виде

$$
p=p(t, x), \quad \rho=g(x), \quad v=\frac{h(t)}{g(x)} .
$$

Одно из этих решений, полученных подстановкой (2.70) в уравнения (2.69), довольно простое и имеет вид:

$$
p=\frac{C}{(a t+l)^{\gamma}}, \quad v=\frac{a x+b}{a t+l}, \quad \rho=\frac{1}{a x+b} .
$$

Второе решение дается следующими формулами:

$$
\begin{gathered}
p=\frac{2(\gamma-1)(a x+b)}{[(\gamma+1) a t+l]^{2}}+K[(\gamma+1) a t+l]^{-2 \gamma /(\gamma+1)}, \\
v=\frac{2(a x+b)}{(\gamma+1) a t+l}, \quad \rho=\frac{1}{a x+b} .
\end{gathered}
$$

Поступая как в доказательстве предложений 2.2, 2.3 и имея в виду замечание 2.2, можно убедиться, что приведенные выше решения не являются ни инвариантными, ни частично инвариантными решениями.

\section{4. Нелинейная диффузия в анизотропных средах}

Уравнение теплопроводности в анизотропной среде без источника имеет вид

$$
u_{t}=\left[f(u) u_{x}\right]_{x}+\left[g(u) u_{y}\right]_{y}+\left[h(u) u_{z}\right]_{z},
$$

где функции $f(u), g(u), h(u)$ положительны согласно своему физическому значению. Аналогичное уравнение с источником имеет вид

$$
u_{t}=\left(f(u) u_{x}\right)_{x}+\left(g(u) u_{y}\right)_{y}+\left(h(u) u_{z}\right)_{z}+q(u) .
$$




\subsection{1. Законы сохранения двумерной модели с источником специ-} ального вида. Двумерное уравнение

$$
u_{t}=\left(f(u) u_{x}\right)_{x}+\left(g(u) u_{y}\right)_{y}+q(u)
$$

с произвольными функциями $f(u), g(u)$ и $q(u) \neq 0$ обладает тремя симметриями, а именно группами переносов по времени и пространственным координатам с генераторами

$$
X_{1}=\frac{\partial}{\partial t}, \quad X_{2}=\frac{\partial}{\partial x}, \quad X_{3}=\frac{\partial}{\partial y} .
$$

Уравнение (2.75) с произвольным источником $q(u)$ не является нелинейно самосопряженным и потому не может быть записано в консервативной форме. В наших работах [8], [9] показано, что при некоторых специальных формах источника $q(u)$ уравнение $(2.75)$ нелинейно самосопряженно. Одна из таких нелинейно самосопряженных форм следующая:

$$
u_{t}=\left(f(u) u_{x}\right)_{x}+\left(g(u) u_{y}\right)_{y}+\omega^{2} \mathscr{F}(u)
$$

где $\omega=$ const и

$$
\mathscr{F}(u)=\int f(u) d u .
$$

Соответственно, уравнение (2.77) обладают нетривиальными законами сохранения и, следовательно, может быть записано в консервативной форме. Мы нашли законы сохранения уравнения (2.77), ассоциированные с симметрией относительно переноса по пространственной переменной $x$ с генератором

$$
X_{2}=\frac{\partial}{\partial x} .
$$

А именно, оператор $X_{2}$ порождает следующие четыре сохраняющихся вектора:

$$
\begin{aligned}
& C^{1}=\sin (\omega x) u, \\
& C^{2}=-\sin (\omega x) f(u) u_{x}+\omega \cos (\omega x) \mathscr{F}(u), \\
& C^{3}=-\sin (\omega x) g(u) u_{y} ; \\
& C^{1}=\cos (\omega x) u, \\
& C^{2}=-\cos (\omega x) f(u) u_{x}-\omega \sin (\omega x) \mathscr{F}(u), \\
& C^{3}=-\cos (\omega x) g(u) u_{y} ; \\
& C^{1}=y \sin (\omega x) u, \\
& C^{2}=-y \sin (\omega x) f(u) u_{x}+\omega y \cos (\omega x) \mathscr{F}(u), \\
& C^{3}=-y \sin (\omega x) g(u) u_{y}+\sin (\omega x) \mathscr{G}(u) ; \\
& C^{1}=y \cos (\omega x) u, \\
& C^{2}=-y \cos (\omega x) f(u) u_{x}-\omega y \sin (\omega x) \mathscr{F}(u), \\
& C^{3}=-y \cos (\omega x) g(u) u_{y}+\cos (\omega x) \mathscr{G}(u),
\end{aligned}
$$

где $\mathscr{F}$ - первообразная функции $f(u)$ (формула (2.78)), а $\mathscr{G}$ - аналогичная первообразная для функции $g(u)$ :

$$
\mathscr{G}(u)=\int g(u) d u
$$


2.4.2. Построение точных решений. Мы воспользуемся здесь законом сохранения

$$
D_{t}\left(C^{1}\right)+D_{x}\left(C^{2}\right)+D_{y}\left(C^{3}\right)=0
$$

для вектора (2.79) для того, чтобы найти частное точное решение уравнения (2.77) с помощью метода законов сохранения. Согласно этому методу, мы заменяем закон сохранения (2.84) следующими более сильными условиями:

$$
D_{t}\left(C^{1}\right)=0, \quad D_{x}\left(C^{2}\right)=0, \quad D_{y}\left(C^{3}\right)=0 .
$$

Подставляя компоненту $C^{1}$ вектора $(2.79)$ в первое уравнение системы $(2.85)$, получаем

$$
D_{t}[\sin (\omega x) u]=\sin (\omega x) u_{t}=0
$$

откуда

$$
u_{t}=0
$$

Теперь подставим компоненту $C^{3}$ вектора (2.79) в третье уравнение системы (2.85) и получим

$$
\left(g(u) u_{y}\right)_{y}=0
$$

С учетом уравнений (2.86) и (2.87), исходное уравнение (2.77) принимает вид

$$
\left(f(u) u_{x}\right)_{x}+\omega^{2} \mathscr{F}(u)=0 .
$$

Из наших построений очевидно, что уравнение (2.88) равносильно второму уравнению системы (2.85). Наша задача - решить переопределенную систему уравнений $(2.86),(2.87)$ и $(2.88)$.

Уравнение (2.86) указывает на то, что мы имеем дело со стационарным решением $u=u(x, y)$.

Теперь решим уравнение (2.88). Для этого введем новую зависимую переменную $z$ по формуле

$$
z=\mathscr{F}(u)
$$

и запишем уравнение $(2.88)$ в виде

$$
z_{x x}+\omega^{2} z=0
$$

Заметим, что последнее уравнение содержит переменную у как параметр. Общее решение уравнения (2.90) имеет вид

$$
z=A_{1}(y) \sin (\omega x)+A_{2}(y) \cos (\omega x) .
$$

С учетом обозначения (2.89) мы получаем решение уравнения (2.88) в следующем неявном виде:

$$
\mathscr{F}(u)=A_{1}(y) \sin (\omega x)+A_{2}(y) \cos (\omega x)
$$


Проверим, что функция $u$, заданная формулой (2.91), удовлетворяет уравнению (2.88). Дифференцируя обе части (2.91) по $x$, получаем

$$
\mathscr{F}^{\prime} u_{x}=\omega\left[A_{1} \cos (\omega x)-A_{2} \sin (\omega x)\right] .
$$

Но поскольку $\mathscr{F}^{\prime}(u)=f(u)$, вышеприведенное уравнение дает

$$
\left(f u_{x}\right)_{x}=-\omega^{2}\left[A_{1} \sin (\omega x)+A_{2} \cos (\omega x)\right] .
$$

Уравнения (2.92) и (2.91) доказывают справедливость уравнения (2.88).

Остается удовлетворить условию (2.87). Дифференцируя обе части (2.91) по $y$, получаем

$$
\mathscr{F}^{\prime} u_{y}=A_{1}^{\prime}(y) \sin (\omega x)+A_{2}^{\prime}(y) \cos (\omega x) .
$$

Так как $\mathscr{F}^{\prime}(u)=f(u)$, то

$$
u_{y}=\frac{1}{f(u)}\left[A_{1}^{\prime}(y) \sin (\omega x)+A_{2}^{\prime}(y) \cos (\omega x)\right] .
$$

Поэтому

$$
g(u) u_{y}=\frac{g(u)}{f(u)}\left[A_{1}^{\prime}(y) \sin (\omega x)+A_{2}^{\prime}(y) \cos (\omega x)\right]
$$

и, следовательно,

$$
\begin{aligned}
\left(g u_{y}\right)_{y}=\frac{g}{f}[ & A_{1}^{\prime \prime}(y) \sin \left(\omega(x)+A_{2}^{\prime \prime}(y) \cos (\omega x)\right] \\
& +\left[A_{1}^{\prime}(y) \sin (\omega x)+A_{2}^{\prime} \cos (\omega x)\right] \frac{f g^{\prime}-g f^{\prime}}{f^{2}} u_{y} .
\end{aligned}
$$

Подставив сюда выражение (2.93) для $u_{y}$, мы запишем уравнение $(2.87)$ в следующем виде:

$$
\begin{aligned}
& A_{1}^{\prime \prime}(y) \sin (\omega x)+A_{2}^{\prime \prime}(y) \cos (\omega x) \\
& \quad+\left(\frac{g^{\prime}}{g}-\frac{f^{\prime}}{f}\right) \frac{1}{f}\left[A_{1}^{\prime}(y) \sin (\omega x)+A_{2}^{\prime} \cos (\omega x)\right]^{2}=0 .
\end{aligned}
$$

Из уравнения (2.94) видно, что возможны два случая: либо

$$
A_{1}^{\prime}(y)=A_{2}^{\prime}(y)=0
$$

либо

$$
A_{1}^{\prime \prime}(y)=A_{2}^{\prime \prime}(y)=0, \quad \frac{g^{\prime}}{g}=\frac{f^{\prime}}{f} .
$$

Из условий (2.95) следует, что уравнение (2.77) имеет следующее точное решение, зависящее только от $x$ :

$$
u=\mathscr{F}^{-1}\left(A_{1} \sin (\omega x)+A_{2} \cos (\omega x)\right),
$$

где $A_{1}, A_{2}$ - произвольные постоянные, а $\mathscr{F}^{-1}$ обозначает обратную функцию для $\mathscr{F}$. 
В случае (2.96) мы делаем вывод, что уравнение (2.77) с линейно зависимыми функциями $f$ и $g$,

$$
g(u)=k f(u), \quad k=\mathrm{const},
$$

имеет следующее точное решение:

$$
u=\mathscr{F}^{-1}\left(\left(a_{1} y+b_{1}\right) \sin (\omega x)+\left(a_{2} y+b_{2}\right) \cos (\omega x)\right),
$$

где $a_{i}, b_{i}$ - произвольные постоянные.

ПримеР 2.1. Пусть $f(u)=1 /\left(1+u^{2}\right)$, тогда предыдущее утверждение состоит в том, что уравнение

$$
u_{t}=\left(\frac{1}{1+u^{2}} u_{x}\right)_{x}+\left(k \frac{1}{1+u^{2}} u_{y}\right)_{y}+\omega^{2} \arctan u
$$

имеет следующее решение:

$$
u=\tan \left(\left(a_{1} y+b_{1}\right) \sin (\omega x)+\left(a_{2} y+b_{2}\right) \cos (\omega x)\right) .
$$

Поступая таким образом со всеми остальными законами сохранения, можно получить другие точные решения уравнений анизотропной теплопроводности.

2.4.3. Самосопряженность и нестационарные решения трехмерной модели с линейным источником. Рассмотрим трехмерное уравнение диффузии с линейным источником:

$$
u_{t}=\left(f(u) u_{x}\right)_{x}+\left(g(u) u_{y}\right)_{y}+\left(h(u) u_{z}\right)_{z}+s u+q_{0}, \quad s, q=\mathrm{const} .
$$

Оно нелинейно самосопряженно, а именно, его сопряженное уравнение

$$
v_{t}+f(u) v_{x x}+g(u) v_{y y}+h(u) v_{z z}+s v=0
$$

выполняется после следующей подстановки:

$$
v=\left(a_{1} x y z+a_{2} x y+a_{3} x z+a_{4} y z+a_{5} x+a_{6} y+a_{7} z+a_{8}\right) \mathrm{e}^{-s t} .
$$

Используя симметрию $X_{2}=\partial / \partial x$, получаем сохраняющийся вектор

$$
\begin{aligned}
& C^{1}=-u v_{x}, \\
& C^{2}=f(u) u_{x} v_{x}+q_{0} v, \\
& C^{3}=g(u) u_{y} v_{x}-G(u) v_{x y}, \\
& C^{4}=h(u) u_{z} v_{x}-H(u) v_{x z} .
\end{aligned}
$$

На основе сохраняющегося вектора (2.102) можно построить точные решения уравнения (2.99). Для этого к уравнению (2.99) нужно добавить следующие дифференциальные связи:

$$
\begin{aligned}
D_{t}\left(u v_{x}\right) & =0, \\
D_{x}\left(f(u) u_{x} v_{x}+q_{0} v\right) & =0, \\
D_{y}\left(g(u) u_{y} v_{x}-G(u) v_{x y}\right) & =0, \\
D_{z}\left(h(u) u_{z} v_{x}-H(u) v_{x z}\right) & =0 .
\end{aligned}
$$


Приведем здесь результат для частного вида уравнения (2.99), когда его первый и третий коэффициенты постоянны: $f(u)=f_{0}, h(u)=h_{0}$, a $g(u)=1 / u$, т. е. для уравнения

$$
u_{t}=f_{0} u_{x x}+\left(\frac{u_{y}}{u}\right)_{y}+h_{0} u_{z z}+s u .
$$

Интегрирование уравнений (2.103) дает следующее частное точное решение уравнения (2.104):

$$
u=\left[\left(A_{1} x+A_{2}\right) z+B_{1} x+B_{2}\right] \mathrm{e}^{s t+\alpha y},
$$

где $A_{1}, A_{2}, B_{1}, B_{2}$ и $\alpha$ - произвольные постоянные. Решение (2.104) описывает бегущую волну в направлении оси $y$, амплитуда который является билинейной функцией от $x, z$. По смыслу термина “бегущая волна" и вида решения (2.104) ясно, что это инвариантное решение относительно однопараметрической группы переносов, порожденной оператором

$$
X=\alpha \frac{\partial}{\partial t}-s \frac{\partial}{\partial y}
$$

в плоскости $t, y$.

Заметим, что уравнение (2.104) допускает пятимерную алгебру Ли с базисом

$$
\begin{gathered}
X_{1}=\frac{\partial}{\partial t}, \quad X_{2}=\frac{\partial}{\partial x}, \quad X_{3}=\frac{\partial}{\partial y}, \\
X_{4}=\frac{\partial}{\partial z}, \quad X_{5}=y \frac{\partial}{\partial y}-2 u \frac{\partial}{\partial u} .
\end{gathered}
$$

Вычисления, подобные тем, которые использовались при доказательстве предложений 2.2 и 2.3, показывает, что (2.106) представляет собой максимальную подалгебру алгебры Ли (2.107), допускаемую решением (2.105). Знания только инвариантности относительно подалгебры (2.106) недостаточно для нахождения решения (2.105).

\section{Глава 3. Приближенная самосопряженность и приближенные законы сохранения}

\section{1. Введение}

В части 4 работы [6] показано, что метод нелинейной самосопряженности может быть распространен на дифференциальные уравнения с малым параметром, что позволяет строить приближенные законы сохранения, используя приближенные симметрии. В данной главе эта возможность иллюстрируется на примере возмущенного уравнения КдФ. Читатель, интересующийся группами приближенных преобразований и их приложениями к дифференциальным уравнениям с малым параметром, может найти компактное изложение материала в [23; гл. 2, 9]. 
Уравнение КдФ будет записываться в виде

$$
u_{t}=u_{x x x}+u u_{x}
$$

и будет рассматриваться возмущенное уравнение

$$
u_{t}-u_{x x x}-u u_{x}-\varepsilon u=0
$$

с малым параметром $\varepsilon$. Уравнение (3.2), а также все дальнейшие вычисления рассматриваются в первом приближении по $\varepsilon$.

Уравнение (3.2) не имеет вида закона сохранения. Вычисление приближенных законов сохранения позволит найти несколько возможностей привести его к такому виду.

\section{2. Приближенно сопряженное уравнение}

Запишем формальный лагранжиан для уравнения (3.2) в виде

$$
\mathscr{L}=v\left[-u_{t}+u_{x x x}+u u_{x}+\varepsilon u\right] .
$$

Тогда

$$
\frac{\delta \mathscr{L}}{\delta u}=v_{t}-v_{x x x}-D_{x}(u v)+v u_{x}+\varepsilon v=v_{t}-v_{x x x}-u v_{x}+\varepsilon v .
$$

Следовательно, уравнение, приближенно сопряженное к уравнению (3.2), имеет вид

$$
v_{t}-v_{x x x}-u v_{x}+\varepsilon v=0 .
$$

\section{3. Приближенная самосопряженность}

Уравнение КдФ является нелинейно самосопряженным со следующей подстановкой:

$$
v=A_{1}+A_{2} u+A_{3}(x+t u) .
$$

Поэтому в случае возмущенного уравнения (3.2) мы ищем подстановку вида

$$
v=A_{1}+A_{2} u+A_{3}(x+t u)+\varepsilon \psi(t, x, u),
$$

удовлетворяющую условию нелинейной самосопряженности

$$
\left.F^{*}\right|_{v=\phi+\varepsilon \psi}=\lambda F
$$

в первом порядке приближения по $\varepsilon$. Здесь через $F$ и $F^{*}$ обозначены левые части уравнений (3.2) и (3.4) соответственно:

$$
F=u_{t}-u_{x x x}-u u_{x}-\varepsilon u, \quad F^{*}=v_{t}-v_{x x x}-u v_{x}+\varepsilon v .
$$

После подстановки выражения (3.5) в уравнение (3.6) члены без $\varepsilon$ исчезают и уравнение (3.6) дает

$$
\lambda=A_{2}+A_{3} t .
$$


Затем мы заменяем комбинацию $u_{t}-u_{x x x}-u u_{x}$ на $\varepsilon u$ в силу уравнения $(3.2)$, сохраняем в (3.6) только линейные члены по $\varepsilon$ и получаем следующее уравнение:

$$
\begin{aligned}
& 3 u_{x x}\left[u_{x} \psi_{u u}+\psi_{x u}\right]+u_{x}\left[u_{x}^{2} \psi_{u u u}+3 u_{x} \psi_{x u u}+3 \psi_{x x u}\right] \\
& \quad-\left[\psi_{t}-\psi_{x x x}-u \psi_{x}+A_{1}+A_{2} u+A_{3}(x+t u)\right]=\left(A_{2}+A_{3} t\right) u
\end{aligned}
$$

Приравнивая в этом уравнении коэффициент при $u_{x x}$ к нулю: $u_{x} \psi_{u u}+\psi_{x u}=0$, получаем

$$
\psi_{u u}=0, \quad \psi_{x u}=0 .
$$

Решение этих уравнений имеет вид

$$
\psi=f(t) u+g(t, x) .
$$

Теперь уравнение (3.7) сводится к следующему:

$$
\left[f^{\prime}(t)-g_{x}(t, x)\right] u+g_{t}(t, x)-g_{x x x}(t, x)+2\left[A_{2}+A_{3} t\right] u+A_{1}+A_{3} x=0 .
$$

Проделав довольно простые вычисления, мы получаем следующие решения последнего уравнения:

$$
g(t, x)=A_{4}-A_{1} t+\left(A_{5}+2 A_{2}-A_{3} t\right) x, \quad f(t)=A_{6}+A_{5} t-\frac{3}{2} A_{3} t^{2} .
$$

Таким образом, доказано следующее утверждение.

ПРЕДЛОЖЕНИЕ 3.1. Возмущенное уравнение КӘФ (3.2) приближенно самосопряженно. Приближенная подстановка (3.5) имеет вид

$$
\begin{aligned}
v \approx A_{1} & +A_{2} u+A_{3}(x+t u) \\
& +\varepsilon\left[\left(A_{6}+A_{5} t-\frac{3}{2} A_{3} t^{2}\right) u+A_{4}-A_{1} t+\left(A_{5}+2 A_{2}-A_{3} t\right) x\right] .
\end{aligned}
$$

\section{4. Приближенные симметрии}

Известно, что алгебра Ли точечных симметрий уравнения КдФ (3.1) является четырехмерной алгеброй с базисом

$$
\begin{gathered}
X_{1}=\frac{\partial}{\partial t}, \quad X_{2}=\frac{\partial}{\partial x}, \quad X_{3}=t \frac{\partial}{\partial x}-\frac{\partial}{\partial u} \\
X_{4}=3 t \frac{\partial}{\partial t}+x \frac{\partial}{\partial x}-2 u \frac{\partial}{\partial u} .
\end{gathered}
$$

Возмущенное уравнение (3.2) наследует симметрии (3.9) в виде следующих приближенных симметрий (см. [23; раздел 9.4]):

$$
\begin{gathered}
X_{1}=\frac{\partial}{\partial t}, \quad X_{2}=\frac{\partial}{\partial x}, \quad X_{3}=t \frac{\partial}{\partial x}-\frac{\partial}{\partial u}+\varepsilon\left(\frac{1}{2} t^{2} \frac{\partial}{\partial x}-t \frac{\partial}{\partial u}\right) \\
X_{4}=3 t \frac{\partial}{\partial t}+x \frac{\partial}{\partial x}-2 u \frac{\partial}{\partial u}-\varepsilon\left[\frac{9}{2} t^{2} \frac{\partial}{\partial t}+3 t x \frac{\partial}{\partial x}-(6 t u+3 x) \frac{\partial}{\partial u}\right] .
\end{gathered}
$$




\section{5. Приближенные законы сохранения}

Теперь мы можем построить приближенные законы сохранения

$$
\left[D_{t}\left(C^{1}\right)+D_{x}\left(C^{2}\right)\right]_{(3.2)} \approx 0
$$

для возмущенного уравнения (3.2), применяя формулу (1.21) к приближенным симметриям (3.10). В результате получаются следующие выражения:

$$
\begin{aligned}
& C^{1}=-W v, \\
& C^{2}=W\left[u v+v_{x x}\right]-v_{x} D_{x}(W)+v D_{x}^{2}(W) .
\end{aligned}
$$

Мы вычислим сохраняющийся вектор (3.12) для оператора $X_{4}$ из (3.10). В этом случае

$$
W=-2 u-3 t u_{t}-x u_{x}+\varepsilon\left(6 t u+3 x+\frac{9}{2} t^{2} u_{t}+3 t x u_{x}\right) .
$$

Мы несколько упростим вычисления, рассмотрев частный случай подстановки $(3.8)$ с $A_{2}=1, A_{1}=A_{3}=\cdots=A_{6}=0$. Тогда

$$
v=u+2 \varepsilon x
$$

Подставляя (3.13), (3.14) в первую компоненту вектора (3.12) и исключая $u_{t}$ с помощью уравнения (3.2), получаем:

$$
\begin{aligned}
C^{1} \approx\left(2 u+3 t u_{t}+x u_{x}\right)(u+2 \varepsilon x)-\varepsilon & \left(6 t u+3 x+\frac{9}{2} t^{2} u_{t}+3 t x u_{x}\right) u \\
=2 u^{2}+3 t u u_{x x x}+3 t u^{2} u_{x}+x u u_{x} & +\varepsilon\left(x u+6 t x u_{x x x}+3 t x u u_{x}\right. \\
& \left.+2 x^{2} u_{x}-3 t u^{2}-\frac{9}{2} t^{2} u u_{x x x}-\frac{9}{2} t^{2} u^{2} u_{x}\right) .
\end{aligned}
$$

Выделив в этом выражении полную производную по $x$, запишем это выражение в виде

$$
\begin{aligned}
C^{1} \approx & \frac{3}{2} u^{2}-3 \varepsilon\left(x u+\frac{3}{2} t u^{2}\right)+D_{x}\left[\frac{1}{2} x u^{2}+t u^{3}-\frac{3}{2} t u_{x}^{2}+3 t u u_{x x}\right. \\
& \left.+\varepsilon\left(2 x^{2} u+\frac{3}{2} t x u^{2}-\frac{3}{2} t^{2} u^{3}-6 t u_{x}+6 t x u_{x x}+\frac{9}{4} t^{2} u_{x}^{2}-\frac{9}{2} t^{2} u u_{x x}\right)\right]
\end{aligned}
$$

Затем мы вычисляем вторую компоненту вектора (3.12), переводим туда $D_{x}(\ldots)$ из $C^{1}$, умножаем полученный вектор $\left(C^{1}, C^{2}\right)$ на $2 / 3$ и получаем:

$$
\begin{aligned}
& C^{1}=u^{2}-\varepsilon\left(2 x u+3 t u^{2}\right) \\
& C^{2}=u_{x}^{2}-\frac{2}{3} u^{3}-2 u u_{x x}+\varepsilon\left(x u^{2}-2 u_{x}+2 x u_{x x}+2 t u^{3}-3 t u_{x}^{2}+6 t u u_{x x}\right) .
\end{aligned}
$$


Вектор (3.16) удовлетворяет приближенному закону сохранения (3.11) в следующем виде:

$$
\begin{aligned}
D_{t}\left(C^{1}\right)+D_{x}\left(C^{2}\right)= & 2 u\left(u_{t}-u_{x x x}-u u_{x}-\varepsilon u\right) \\
& -2 \varepsilon(x+3 t u)\left(u_{t}-u_{x x x}-u u_{x}\right)+o(\varepsilon) .
\end{aligned}
$$

Это уравнение может быть записано в принятом нами первом приближении в более удобном виде

$$
D_{t}\left(C^{1}\right)+D_{x}\left(C^{2}\right) \approx[2 u-2 \varepsilon(x+3 t u)]\left(u_{t}-u_{x x x}-u u_{x}-\varepsilon u\right) .
$$

Уравнение (3.17) показывает, что возмущенное уравнение КдФ (3.2) может быть записано в виде приближенного закона сохранения

$$
D_{t}\left(C^{1}\right)+D_{x}\left(C^{2}\right) \approx 0
$$

где $C^{1}$ и $C^{2}$ заданы формулами (3.16).

ЗАмечАние 3.1. Зная первую компоненту $C^{1}$ вектора (3.16), его вторую компоненту $C^{2}$ можно вычислить и не обращаясь к его выражению, данному в общих формулах (3.12). Действительно, по построению, $C^{1}$ удовлетворяет уравнению (3.11). Поэтому $\left[D_{t}\left(C^{1}\right)\right]_{(3.2)}$ будет полной производной по $x$ некоторого выражения. Вычислив это выражение, найдем $C^{2}$. Для иллюстрации применим такой подход к $C^{1}$ из (3.16). Имеем:

$$
D_{t}\left(C^{1}\right)=D_{t}\left[u^{2}-\varepsilon\left(2 x u+3 t u^{2}\right)\right]=2 u u_{t}-\varepsilon\left(2 x u_{t}+3 u^{2}+6 t u u_{t}\right) .
$$

Заменив здесь $u_{t}$ его выражением, данным уравнением (3.2), легко получим:

$$
\begin{aligned}
{\left[D_{t}\left(C^{1}\right)\right]_{(3.2)} \approx } & -D_{x}\left[u_{x}^{2}-\frac{2}{3} u^{3}-2 u u_{x x}\right. \\
& \left.+\varepsilon\left(x u^{2}-2 u_{x}+2 x u_{x x}+2 t u^{3}-3 t u_{x}^{2}+6 t u u_{x x}\right)\right]
\end{aligned}
$$

Выражение в квадратных скобках совпадает с $C^{2}$ из (3.16).

Применим предложенную в замечании 3.1 процедуру для вычисления сохраняющегося вектора (3.12), соответствующего оператору $X_{4}$, взяв частный случай подстановки $(3.8)$ с $A_{1}=1, A_{2}=A_{3}=\cdots=A_{6}=0$. Тогда мы имеем

$$
v=1-\varepsilon t
$$

и, используя первое уравнение из (3.12), получаем

$$
C^{1}=u-\varepsilon t u \text {. }
$$

Следуя нашей процедуре, последовательно находим

$$
D_{t}\left(C^{1}\right)=D_{t}(u-\varepsilon t u)=u_{t}-\varepsilon\left(u+t u_{t}\right)
$$


и

$$
\left[D_{t}\left(C^{1}\right)\right]_{(3.2)} \approx(1-\varepsilon t)\left(u_{x x x}+u u_{x}\right)=(1-\varepsilon t) D_{x}\left(u_{x x}+\frac{1}{2} u^{2}\right) .
$$

Значит,

$$
C^{2}=-(1-\varepsilon t)\left(u_{x x}+\frac{1}{2} u^{2}\right) .
$$

Мы получили сохраняющийся вектор

$$
C^{1}=(1-\varepsilon t) u, \quad C^{2}=-(1-\varepsilon t)\left(u_{x x}+\frac{1}{2} u^{2}\right) .
$$

Он удовлетворяет приближенному закону сохранения (3.11) в следующем виде:

$$
D_{t}\left(C^{1}\right)+D_{x}\left(C^{2}\right) \approx(1-\varepsilon t)\left(u_{t}-u_{x x x}-u u_{x}-\varepsilon u\right) .
$$

Таким образом, в дополнение к уравнению (3.19) мы получили еще одно представление возмущенного уравнения КдФ (3.2) в виде приближенного закона сохранения:

$$
D_{t}[(1-\varepsilon t) u]+D_{x}\left[-(1-\varepsilon t)\left(u_{x x}+\frac{1}{2} u^{2}\right)\right] \approx 0 .
$$

Наличие в подстановке (3.8) шести независимых постоянных $A_{1}, \ldots, A_{6}$ позволяет построить для оператора $X_{4}$ шесть различных представлений возмущенного уравнения КдФ (3.2) в виде приближенного закона сохранения. То же самое можно проделать для остальных трех операторов из (3.10).

Мы благодарим неизвестного нам рецензента за ценные замечания и конструктивные предложения, которые были учтены при подготовке окончательного варианта статьи.

\section{Список литературы}

[1] S. Lie, Gesammelte Abhandlungen, v.1-6, B. G. Teubner, Leipzig, 1922-1937, xix +862 , ix+988, xvi+789, xii+684, xii+776, xxiv+940 pp.

[2] Л. В. Овсянников, Групповые свойства дифференциальных уравнений, Изд-во СО АН СССР, Новосибирск, 1962, 239 с.

[3] Л.В. Овсянников, Лекции по теории групповых свойств дифференииальных уравнений, Изд-во Новосибирского гос. ун-та, Новосибирск, 1966, 131 с.

[4] N. H. Ibragimov, "A new conservation theorem", J. Math. Anal. Appl., 333:1 (2007), 311-328.

[5] N.H. Ibragimov, "Nonlinear self-adjointness in constructing conservation laws", Archives of ALGA, 7/8 (2010-2011), 1-99; arXiv: 1109.1728v1.

[6] N. H. Ibragimov, "Nonlinear self-adjointness and conservation laws", J. Phys. A, 44:43 (2011), 432002, 8 pp.

[7] N. H. Ibragimov, "Method of conservation laws for constructing solutions to systems of PDEs", Discontinuity, Nonlinearity, and Complexity, 1:4 (2012), 353-365.

[8] N. H. Ibragimov, E. D. Avdonina, "Heat conduction in anisotropic media", Discontinuity, Nonlinearity and Complexity, 1:3 (2012), 237-251. 
[9] Н. Х. Ибрагимов, Е. Д. Авдонина, "Построение точных решений уравнений анизотропной теплопроводности с помощью законов сохранения", Материаль V Pocсийской конферениии с международным участием "Многофазные системы: теория и приложения" (Уфа, 2012), Часть 1, Тр. Ин-та мех. УНЦ РАН, 9, 2012, 88-90.

[10] E. D. Avdonina, N.H. Ibragimov, R. Khamitova, "Exact solutions of gasdynamic equations obtained by the method of conservation laws", Commun. Nonlinear Sci. Numer. Simul., 18:9 (2013), 2359-2366.

[11] E. D. Avdonina, N. H. Ibragimov, "Conservation laws and exact solutions for nonlinear diffusion in anisotropic media", Commun. Nonlinear Sci. Numer. Simul., 18:10 (2013), 2595-2603.

[12] Н.Х. Ибрагимов, Группь преобразований в математической физике, Наука, M., 1983, 280 с.; англ. пер.: N.H. Ibragimov, Transformation groups applied to mathematical physics, Math. Appl. (Soviet Ser.), D. Reidel Publishing Co., Dordrecht, 1985, xv+394 pp.

[13] Л.В. Овсянников, Групповой анализ дифференциалънъх уравнений, Наука, М., 1978, 399 с.; англ. пер.: L. V. Ovsiannikov, Group analysis of differential equations, Academic Press, Inc. [Harcourt Brace Jovanovich, Publishers], New York-London, 1982, xvi+416 pp.

[14] П. Олвер, Приложения групп Ли к дифференииальным уравнениям, Мир, М., 1989, 639 с.; пер. с англ.: Р. J. Olver, Applications of Lie groups to differential equations, Grad. Texts in Math., 107, Springer-Verlag, New York, 1986, xxvi+497 pp.

[15] Р. Курант, Д. Гильберт, Методъ математической физики, т. 1, Гостехиздат, M., 1933, 525 с.; пер. с нем.: R. Courant, D. Hilbert, Methoden der mathematischen Physik, v. I, Die Grundlehren der mathematischen Wissenschaften in Einzeldarstellungen, 12, Springer, Berlin, 1924, xiii+450 pp.

[16] N.H. Ibragimov, Elementary Lie group analysis and ordinary differential equations, Wiley Ser. Math. Methods Pract., 4, John Wiley \& Sons, Ltd., Chichester, 1999, $\mathrm{xviii}+347 \mathrm{c}$.

[17] Б. Б. Кадомцев, В. И. Петвиашвили, "Об устойчивости уединенных волн в слабодисперсионных средах", Докл. АН СССР, 192 (1970), 753-756; англ. пер.: B. B. Kadomtsev, V.I. Petviashvili, "On the stability of solitary waves in weakly dispersing media", Soviet Phys. Dokl., 15 (1970), 539-541.

[18] В.Е. Захаров, С. В. Манаков, С. П. Новиков, Л. П. Питаевский, Теория солитонов: Метод обратной задачи, Наука, М., 1980, 320 с.; англ. пер.: S. Novikov, S. V. Manakov, L.P. Pitaevskii, V.E. Zakharov, Theory of solitons. The inverse scattering method, Contemp. Soviet Math., Consultants Bureau [Plenum], New York, 1984, xi+276 pp.

[19] T. Schäfer, C. E. Wayne, "Propagation of ultra-short optical pulses in cubic nonlinear media", Phys. D, 196:1-2 (2004), 90-105.

[20] A. Sakovich, S. Sakovich, "The short pulse equation is integrable", J. Phys. Soc. Japan, 74:1 (2005), 239-241.

[21] A. Sakovich, S. Sakovich, "Solitary wave solutions of the short pulse equation", J. Phys. A, 39:22 (2006), L361-L367.

[22] И.Ш. Ахатов, Р. К. Газизов, Н. Х. Ибрагимов, "Нелокальные симметрии. Эвристический подход", Итоги науки и техники. Сер. Соврем. пробл. матем. Нов. достиж., 34, ВИНИТИ, М., 1989, 3-83; англ. пер.: I. Sh. Akhatov, R. K. Gazizov, N. Kh. Ibragimov, "Nonlocal symmetries. Heuristic approach", J. Soviet Math., 55:1 (1991), 1401-1450. 
[23] N. H. Ibragimov (ed.), CRC handbook of Lie group analysis of differential equations, v. 3: New trends in theoretical developments and computational methods, CRC Press Inc., Boca Raton, FL, 1996, xvi+536 pp.

\section{H. Х. Ибрагимов (N. H. Ibragimov)}

Уфимский государственный авиационный технический университет;

Blekinge Institute of Technology, Karlskrona, Sweden

E-mail: nailhib@mail.ru

Е. Д. Авдонина (E. D. Avdonina)

Уфимский государственный авиационный технический университет

E-mail: alenaish@yahoo.com
Поступила в редакцию 14.12 .2012 